\title{
Nanopatterns with Biological Functions
}

\author{
Thomas Blättler ${ }^{1, \dagger}$, Christoph Huwiler ${ }^{1, \dagger}$, Mirjam Ochsner ${ }^{1, \dagger}$, Brigitte Städler ${ }^{1, \dagger}$, \\ Harun Solak ${ }^{2}$, Janos Vörös ${ }^{1}$, and H. Michelle Grandin ${ }^{1, *}$ \\ ${ }^{1}$ Biolnterface Group, Laboratory for Surface Science and Technology, Department of Materials, ETH Zurich, Switzerland \\ ${ }^{2}$ Laboratory for Micro- and Nanotechnology, Paul Scherrer Institute, Villigen, Switzerland
}

\begin{abstract}
Both curiosity and a desire for efficiency have advanced our ability to manipulate materials with great precision on the micrometer and, more recently, on the nanometer scale. Certainly, the semiconductor and integrated circuit industry has put the pressure on scientist and engineers to develop better and faster nanofabrication techniques. Furthermore, our curiosity as to how life works, and how it can be improved from a medical perspective, stands to gain a great deal from advances in nanotechnology. Novel nanofabrication techniques are opening up the possibilities for mimicking the inherently nano-world of the cell, i.e., the nanotopographies of the extracellular matrix (ECM) and the nanochemistry presented on both the cell membrane and the ECM. In addition, biosensing applications that rely on fabrication of high-density, precision arrays, e.g., DNA or gene chips and protein arrays, will gain significantly in efficiency and, thus, in usefulness once it becomes possible to fabricate heterogeneous nanoarrays. Clearly, continued advances in nanotechnology are desired and required for advances in biotechnology. In this review, we describe the leading techniques for generating nanopatterns with biological function including parallel techniques such as extreme ultraviolet interference lithography (EUV-IL), soft-lithographic techniques (e.g., replica molding (RM) and microcontact printing $(\mu \mathrm{CP})$ ), nanoimprint lithography (NIL), nanosphere lithography (NSL) (e.g., colloid lithography or colloidal block-copolymer micelle lithography) and the nanostencil technique, in addition to direct-writing techniques including e-beam lithography (EBL), focused ion-beam lithography (FIBL) and dip-pen nanolithography (DPN). Details on how the patterns are generated, how biological function is imparted to the nanopatterns, and examples of how these surfaces can and are being used for biological applications will be presented. This review further illustrates the rapid pace by which advances are being made in the field of nanobiotechnology, owing to an increasing number of research endeavors, for an ever increasing number of applications.
\end{abstract}

Keywords: Nanopatterns, Biological Functions, Lithography.

\section{CONTENTS}

1. Introduction ........................... 2237

1.1. Importance of Micron Scale Patterning ............ 2237

1.2. Motivations for Going Nano . . . . . . . . . . . . . . 2239

1.3. Concept of Nanobiopatterning . . . . . . . . . . . . . 2239

1.4. Content of this Review ..................... 2240

2. Parallel Nanopatterning Techniques ................ 2240

2.1. Optical Nanolithography $\ldots \ldots \ldots \ldots \ldots \ldots \ldots \ldots 2241$

2.2. Microcontact Printing. .................... 2242

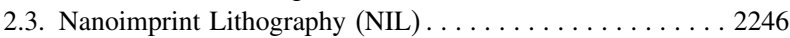

2.4. Colloid Lithography ...................... 2248

2.5. Block-Copolymer Micelle Lithography . . . . . . . . . 2251

2.6. Nanostencil (Shadow Mask Lithography). . . . . . . . . . 2253

3. Serial Nanopatterning Techniques ................. 2254

3.1. E-beam and Focused Ion-Beam Lithography . . . . . . . . 2254

3.2. Dip-Pen Nanolithography (DPN) . . . . . . . . . . . . . . . 2254

4. Conclusions and Outlook..................... 2260

*Author to whom correspondence should be addressed.

${ }^{\dagger}$ These authors have contributed equally to the article.
Acknowledgments . . . . . . . . . . . . . . . . . . 2260

References and Notes . . . . . . . . . . . . . . . . . . . . . . . 2260

\section{INTRODUCTION}

\subsection{Importance of Micron Scale Patterning}

The technology platform of microfabrication, developed primarily for and by the semiconductor industry, has since become a useful tool in the emerging fields of biotechnology for generating patterns on surfaces in the micron range. ${ }^{1-3}$ The field of genomics, for example, has benefited greatly from DNA microarrays for high-throughput identification of genes and genetic mutations in healthy and diseased tissue. ${ }^{4,5}$ Such microarrays are also being developed for protein ${ }^{6,7}$ sugar, ${ }^{8}$ and cell ${ }^{9-11}$ based drug development and diagnostic screening applications. In the fields of synthetic and analytical chemistry, microstructured surfaces have enabled new combinatorial methods of synthesis and 

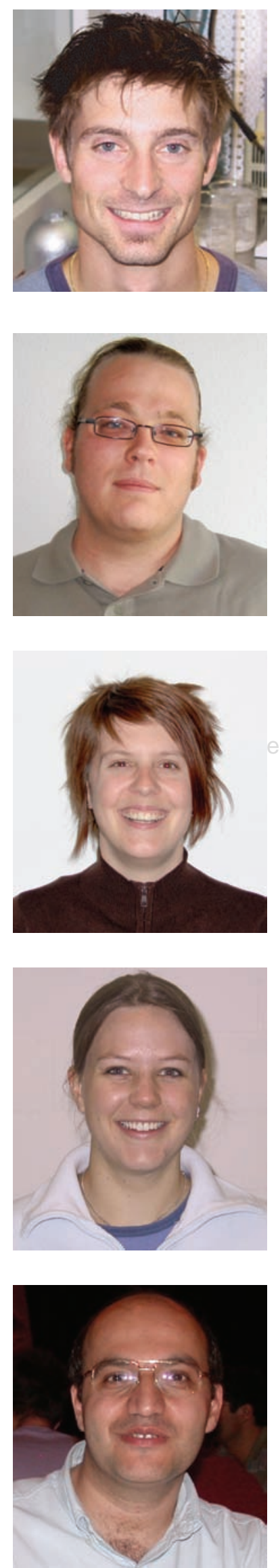

Harun H. Solak received his Ph.D. in Electrical and Computer Engineering from the University of Wisconsin-Madison in 1998. His thesis work was on using X-ray microscopy techniques to investigate physical mechanisms responsible for failure of integrated circuits. He performed his experiments at a number of synchrotron radiation laboratories in the U.S. In his postdoctoral work at the same university he demonstrated the use of coherent synchrotron radiation in the extreme ultraviolet range to print nanometer scale periodic structures. He has been a staff scientist at the Paul Scherrrer Institute since 2001 where he set up a facility for EUV lithography. His research interests include nanofabrication techniques, guided self-assembly, X-ray optics, and nano-photonics.

Thomas M. Blättler received his M.Sc. in 2004 from the materials department of the ETH Zurich, Switzerland. During his M.Sc. thesis at the University of South Australia (group of Hans Griesser) he investigated a covalent approach to immobilize selected poly(ethylene glycol) (PEG) molecules. He is currently working on his Ph.D. in the Laboratory for Surface Science and Technology, ETH Zurich, Switzerland. His current research interests focus on nanopatterning in the field of bio-interfaces with the aim to apply these patterns for single protein investigations. More specifically, he is using particle self-assembly routes as templates to produce nanopatterned substrates or to produce replicas in polymers (nanocavities). Such patterned surfaces or cavities are of high interest to investigate single molecules after further PEG modifications. To specifically modify and functionalize the created patterns he is now also using microfluidic devices.

Christoph B. Huwiler received his M.Sc. in 2001 from the materials department of the ETH Zurich, Switzerland. During his M.Sc. thesis at the ETH in Zurich (in the group of Professor Gauckler) he investigated the sintering and grain growth behavior of doped ceria and ceria-gadolinia nanopowders and their possible use as electrolyte materials in solid oxide fuel cells. Currently, he is finishing his Ph.D. in the Laboratory for Surface Science and Technology and the Laboratory of Biosensors and Bioelectronics, both at the ETH Zurich, Switzerland. He is interested in the self-assembly of colloidal nano-particles and the development of functional nano-particle arrays, with a special focus on their use in biosensing applications as well as on the fundamental factors that govern nanoparticle self-assembly and adsorption on structured surfaces.

Mirjam Ochsner received her M.Sc. in 2005 from the materials department of the ETH Zurich, Switzerland. During her M.Sc. she was working in the field of solar cells in the Chemical Physics Group at Chalmers University of Technology in Göteborg, Sweden. She is currently working on her Ph.D. in the Laboratory for Surface Science and Technology, ETH Zurich, Switzerland. Her current research interests focus on the field of bio-interfaces, especially on engineered surfaces for micro-3-D cell culturing, with the aim to produce a set of tools which combine 2-dimensional chemical patterning with topographical microstructuring, thus presenting to the cells a controlled micro-environment that mimics the in vivo environment. This allows her to culture single cells in an array format and to control the three dimensional shape of each individual cell.

Brigitte M. Städler received her M.Sc. in 2004 from the materials department of the ETH Zurich, Switzerland. During her M.Sc. thesis at the Chalmers University in Gothenburg, Sweden (group of Bengt Kasemo) she investigated the micropatterning of DNA-tagged vesicles. She is currently working on her Ph.D. in the Laboratory for Surface Science and Technology, ETH Zurich, Switzerland. Her current research interests focus on nano structuring surfaces. More specifically, she combines a high-tech nanopatterning method and the self-assembly capability of DNA in order surface immobilized gold colloids or vesicles. 


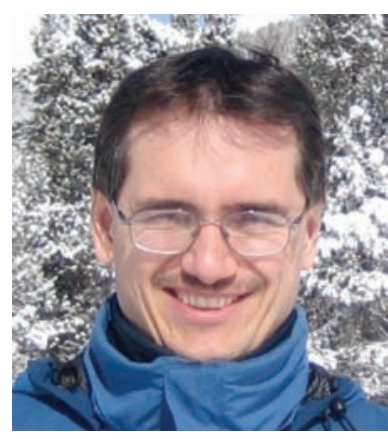

Janos Vörös received a M.S. degree in physics and a Ph.D. in biophysics from the Eötvös University in Budapest, Hungary. From 1998 until 2005 he worked in the BioInterface Group of the Laboratory for Surface Science and Technology, ETH Zurich. Currently he is professor of bioelectronics leading the Laboratory of Biosensors and Bioelectronics at the Institute for Biomedical Engineering of ETH Zurich, Switzerland. He is interested in the understanding and controlling of cellular and biomolecular processes at the nanoscale by physical and chemical means. His research covers aspects such as the creation of nanopatterned non-fouling surfaces with functional arrays of macromolecules, the development of novel experimental techniques for the study of molecular and cellular interactions, the dynamic control of macromolecule-surface interaction by electronic means for applications in neurobiology and the use of (micro/nano/bio)technology in proteomics and tissue engineering. Vörös can be reached by e-mail at janos.voros@biomed.ee.ethz.ch.

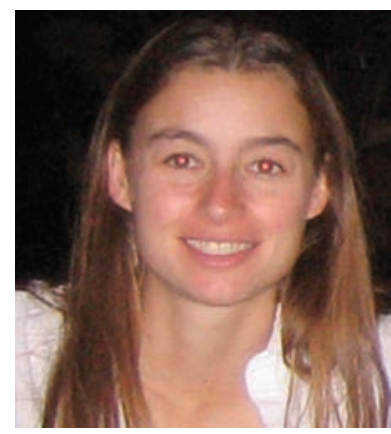

H. Michelle Grandin received her Ph.D. in Chemistry from the University of Western Ontario, Canada where she studied the interface between thin-films of metals and organic semiconductors pertaining to Organic Light Emitting Diodes, under the supervision of Professor P. Norton. She is currently working as a senior postdoctoral fellow in the BioInterface Group of Professor M. Textor in the Laboratory for Surface Science and Technology at the ETH Zurich, Switzerland. Her current research interests focus on the development of novel evanescent-field-based sensing techniques for investigating the biointerface in-situ and in-real time, with applications ranging from single molecule detection, to the study of supported lipid bilayer formation, as well as cell-surface interactions. She is also working toward chemical and physical patterning platforms for the controlled study of cellular behavior including polarization and mechanotransduction.

microbiochemical assays. ${ }^{12-14}$ Furthermore, in the fields of biology and implantology, fundamental studies of cellsurface and cell-extracellular matrix interactions have also benefited from micropatterned surfaces by which control over adhesion ligand presentation (type, density, and spatial distribution), cell geometry, and local substrate compliance have been shown to affect cell behavior. ${ }^{15-18}$ In addition, micropatterns have been used to guide cell growth with application for neuronal implants, in-vitro neural networks and other tissue engineering applications. ${ }^{19-22}$

\subsection{Motivations for Going Nano}

However, much as the semiconductor industry is not satisfied with the density of devices attainable for integrated circuits using microfabrication and as they are now seeking out new methods for nanofabrication, biotechnology, and fundamental biology also stands to gain significantly by 'going nano'. The advantages for gene-chips and other biosensors are much the same, namely an increase in aerial density of the probe materials, be it DNA or otherwise, by several orders of magnitude. ${ }^{23,24}$ In this way, screening a correspondingly larger number of targets, or the same number of targets from smaller sample volumes, becomes possible. In fact, nanoarrays could enable screening the entire human genome on a single chip measuring $2 \times 2 \mathrm{~cm}^{2}$ of $150 \mathrm{~nm}$ spot sizes, versus 10000 of today's state of the art chips with $20 \mu \mathrm{m}$ spot sizes. ${ }^{23}$

Nanofabrication also promises to enhance our ability to understand the fundamentals of cell behavior by mimicking the inherently nano-world of the cell from the nanotopographies of the extracellular matrix (ECM) to the nanochemistry presented on both the cell membrane and the ECM. As the size of features produced by nanobiotechnology approaches the length-scale of nature's individual building blocks, i.e., the size of proteins, novel questions can be asked and new tools arise to address them. At the nanoscale, chemistry and topography are inherently inseparable since proteins use both to interact with each other and to form supramolecular complexes via spatially distributed, multivalent interactions commonly referred to as lock and key mechanisms. Indeed, both nanoscale topography ${ }^{25-28}$ and chemistry ${ }^{29,30}$ have already been shown to affect cell behavior.

Additionally, nanofabrication of nanoelectrode arrays is being pursued in the field of neuronal electrophysiology in order to increase sampling capabilities. ${ }^{31}$ Nanoelectronics combined with the ability to control single molecules on the nanoscale will also enable further development in the field of molecular electronics. ${ }^{32,33}$ Furthermore, studies of single biomolecules, ranging from DNA to proteins and enzymes, will be advanced significantly through the ability to immobilize single molecules onto nanopatterns with micron spacing, thereby making them amenable to microscopy based detection, wherein fluorescence is most commonly used to study conformational studies and single binding events.

\subsection{Concept of Nanobiopatterning}

In moving fabrication from the micron to the nanorange, it has become clear that new techniques are required since the conventional technique for microfabrication, i.e., photolithography is constrained by the diffraction limit of 
light. Correspondingly, a vast number of new technologies are being explored as candidates for nanoscale patterning platforms. ${ }^{23,34-40}$ Furthermore, in adopting any technology from the realm of silicon to the realm of proteins and other biomolecules, many aspects of the technique need to be modified, such as working with the aqueous environment that accompanies biology. $2,41,42$

The vast majority of biological interactions are based on highly selective and specific interactions. In contrast, uncontrolled, non-specific interactions are usually the cause of device-failure in a biological environment. For example, the rejection of an implant due to immune reactions, the short circulation time of drug-delivery particles, and the false response of biosensors are often caused by the non-specific adsorption of proteins. As such, incorporating chemical contrast onto the surface for applications in biotechnology requires the ability to define areas of specific interactions in a non-interacting background. Biology chose a combination of lipids, sugars, and proteins (i.e., the cellular membranes) as the non-interactive, mobile, and highly hydrated surface at which most bioprocesses take place. Although several groups have managed to mimic this strategy using lipid based micro- and nanopatterns, ${ }^{43-47}$ it remains, at present, that approaches utilizing the excellent protein resistant properties and stability of poly(ethylene glycol) (PEG) coatings are generally more compatible with the processing steps of current nanotechnology methods. Various techniques exist to attach PEG to surfaces, e.g., using end-grafting methods, ${ }^{48-50}$ block copolymers such as pluronics ${ }^{51}$ self-assembled monolayers,${ }^{52}$ interpenetrating networks, ${ }^{53}$ and the spontaneous adsorption of polyelectrolyte grafted PEG. ${ }^{54}$

After eliminating the non-specific interactions with a surface, different nanopatterning methods can be used to introduce selected biofunctionalities onto well defined spots of the substrate, in an otherwise 'silent' background. Proteins, DNA, sugars, or short peptide sequences are commonly introduced onto surfaces to elicit specific cellular responses or to capture specific ligands from a complex biological sample. The nanobiopatterning methods, discussed in the next sections, aim to control the location, distribution, orientation, and conformation of these bioligands on the nanometer scale. It should be noted that, for the purpose of this review, we shall refer to nanobiotechnology as a field wherein the tools of nanotechnology are used to create biofunctional nanopatterns for biological applications. The inverse field of bionanotechnology in which biology is used to create nanopatterns, generally for inorganic applications, although very interesting in its own right, is beyond the scope of this review. For a discussion of topics in bionanotechnology, the reader is referred to the following references. ${ }^{55,56}$

\subsection{Content of this Review}

Therefore, the aim of this review is to outline the most promising techniques of nanofabrication that are being developed for biological applications. We distinguish between parallel and serial approaches of nanofabrication, whereby the former enables the fast production of a large number of samples and can be used over large surface areas (parallel nanopatterning techniques, Section 2), while the latter techniques, though slower, often offer greater control and smaller feature sizes (serial nanopatterning techniques, Section 3). Often, so called top-down techniques are combined with bottom-up approaches, such as the self-assembly of alkanethiols or phosphates to provide further flexibility in addressing the nanorange. ${ }^{57}$ Also to be addressed in this review, are the means and conditions by which biological function is incorporated into nanopatterns. In addition, several specific examples, illustrating the usefulness of nanopatterns in addressing biological questions, will be presented. And finally, the advantages and limitations of each technique will be compared in the context of relevant applications. In so doing, we hope to illustrate the impact that advances in nanobiotechnology can and are making for applications ranging from biosensors and implants to tissue engineering, as well as in fundamental biology.

\section{PARALLEL NANOPATTERNING TECHNIQUES}

Currently, the ability to produce well-defined nanopatterns remains a challenging goal in both the semiconductor industry and the field of biotechnology. On the one hand, reproducible high quality patterns are required for various applications, but on the other hand, the time and price to achieve them has to be taken into consideration. For industrial scale production, large areas must be patterned at a reasonably low price in a reasonably short time. Correspondingly, a number of novel parallel nanopatterning methods are being developed including the next generation of photolithography (e.g., extreme ultraviolet interference lithography (EUV-IL)), soft-lithographic techniques (e.g., replica molding (RM) and microcontact printing $(\mu \mathrm{CP}))$, nanoimprint lithography (NIL), nanosphere lithography (NSL) (e.g., colloid lithography or colloidal block-copolymer micelle lithography), and the nanostencil technique. Apart from nanosphere lithography, all these techniques require a mask or master generated by serial writing (focused ion or $e$-beam lithography) which is generally the rate limiting step in producing nanopatterns by parallel techniques. ${ }^{34,58}$ Fortunately, these masks and masters can be reused many times for replication, which is a particular advantage when many identical samples are needed as example for cell studies. However, when new features are needed, a new mask must be produced. In general, most parallel patterning techniques are limited to flat substrates. $\mu \mathrm{CP}$, however, can be applied on rough or non-flat surfaces, which might turn out to be useful if patterning of implants is required. Each of the aforementioned techniques has the potential for low-cost, large 
scale production of nanopatterns. The specific advantages and limitations of each technique will be described in more detail in what follows, with a particular emphasis on highlighting their uses and potential uses for various bioapplications.

\subsection{Optical Nanolithography}

Optical lithography is probably the most widely used and best established lithography technique available. This is primarily due to the semiconductor industry which uses this method to create highly integrated circuits in the micrometer range. The need for faster and smaller electronic devices continues to push the resolution limits of the features towards smaller scales. The Rayleigh equation defines the resolution limit of conventional projection optical lithography as a function of wavelength and numerical aperture. This fact opens up two possibilities to increase the resolution; using lens systems with higher numerical aperture and/or decreasing the wavelengths. ${ }^{59}$

Conventional projection optical lithography using ultraviolet (UV) light has already demonstrated that it can be used to create biologically relevant micropatterns via an etching or a lift-off process, for example. ${ }^{60,61}$ Holden et al. have also demonstrated a way to photo-attach biotin-4-fluorescein to a surface without the need of UV light in order to create micropatterns of enzymes in a microfluidic channel. ${ }^{62}$ In the submicron range, there are a few approaches which make use of UV light to pattern biomolecules with the help of interference. ${ }^{63-65}$ In the nanorange, we have recently developed an approach to generate biofunctional nanopatterns using optical lithography as described below.

\subsubsection{Extreme Ultraviolet Interference Lithography (EUV-IL)}

Interference lithography (IL) is, in fact, commonly used in combination with UV-light for making high density periodic patterns over large areas but it is limited in pattern resolution by the wavelength. In 1999, Solak et al., ${ }^{36}$ showed that combining IL and the extreme ultraviolet (EUV) light from undulators at synchrotron radiation facilities with an interferometer based on reflective optics (mirrors) allows for the creation of features in a photoresist material with a period of $38 \mathrm{~nm}$. The available EUV light sources emit radiation with rather large spectral width $(2-4 \%)$ that directly limits the number of periods produced with the reflective optics based interferometer. However, by using achromatic interferometers, based on transmission diffraction gratings, these difficulties have been overcome. $141 \mathrm{~nm}$ period arrays of holes were created by applying a multi beam scheme using diffraction gratings fabricated by e-beam lithography. ${ }^{66}$ The geometry for making one-dimensional (lines) and two-dimensional (dots) grating patterns are shown in Figures $1 \mathrm{~A}$ and 1B,
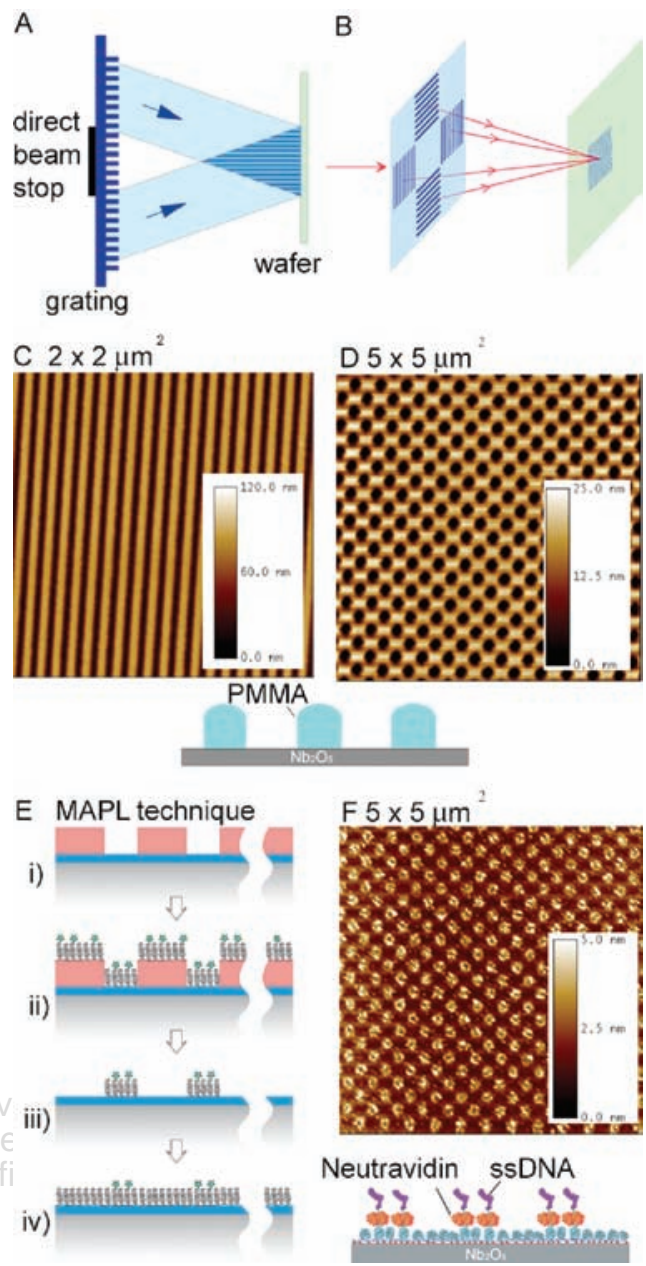

Fig. 1. Set up to create lines (A) and dots (B) by two-beam and fourbeam interference, respectively. Reprinted with permission from [66], H. H. Solak et al., Microelectronic Engineering 67-8, 56 (2003). () 2003. The corresponding tapping mode AFM images in air of a nanopatterned PMMA resist layer are shown (Size: $50 \mathrm{~nm}$ lines (C), dot diameter $200 \mathrm{~nm}$ in a distance of $200 \mathrm{~nm}$ (D)). (E) Molecular Assembly Patterning by Lift-off (MAPL). A pre-patterned resist coated surface (i), is dipped into a solution of PLL- $g$-PEG/PEG-Y ( $\mathrm{Y}=\mathrm{a}$ bio-specific ligand) (ii), followed by lift-off of the residual resist in an organic solvent (iii), and finally backfilling of the background with bio-resistant PLL-g-PEG (iv). Reprinted with permission from [60], D. Falconnet et al., Adv. Funct. Mater. 14, 749 (2004). (C) 2004, Wiley-VCH. (F) Shows the transfer of a PMMA- $\mathrm{Nb}_{2} \mathrm{O}_{5}$ structure into a biologically relevant pattern of spots of surface immobilized complexes of single stranded DNA (ssDNA) and neutravidin in a bio-resistant background of PLL- $g$-PEG via the MAPL technique.

respectively. ${ }^{67}$ Tapping mode AFM images of the PMMA resist $/ \mathrm{Nb}_{2} \mathrm{O}_{5}$ oxide patterns obtained using these schemes are shown in Figures 1C and 1D, respectively. Chemical patterns of nanolines created by EUV-IL have been used as a template to induce epitaxial self-assembly of domains of a block copolymer when the period of the nanolines matched the lamellar period of the block copolymer. ${ }^{68}$

Furthermore, biological function can be incorporated into these nanopatterns using the Molecular Assembly Patterning by Lift-off (MAPL) technique, developed in our 
group ${ }^{60}$ The MAPL technique is a method which creates micropatterns via standard photolithography and the assembly of bio-functionalized PLL- $g$-PEG. PLL- $g$-PEG consists of a positively charged poly(L-lysine) (PLL) backbone to which poly(ethylene glycol) (PEG) chains are grafted. This polymer is known to resist protein adsorption almost completely and furthermore offers the possibility to functionalize the PEG chains with specific bioreceptors (e.g., biotin, peptide sequences) ${ }^{69,70}$ In MAPL, a resist modified oxide surface (e.g., $\mathrm{Nb}_{2} \mathrm{O}_{5}, \mathrm{TiO}_{2}$, or $\left.\mathrm{SiO}_{2}\right)($ Fig. $1 \mathrm{E}(\mathrm{i}))$ is dipped into a solution containing the functionalized PLL- $g$-PEG/PEG-Y ( $\mathrm{Y}=$ a bio-specific ligand) (Fig. 1E(ii)), then the resist is removed in an organic solvent (Fig. 1E(iii)) and finally, the background is backfilled with the non-functionalized bio-resistant PLL$g$-PEG (Fig. 1E(iv)). In the micrometer range MAPL has been successfully applied to cell studies by using RGDfunctionalized PLL-g-PEG in the active patches, whereby RGD is a tripeptide sequence known to specifically bind to the integrin receptors found in the cell membrane. The spots can be further functionalized via the biotin/ avidin linkage (and other strategies ${ }^{71}$ ) if PLL- $g$-PEG/PEGbiotin $^{60,72}$ is immobilized.

Recent experiments performed in our group show the feasibility of transferring PMMA resist/ $/ \mathrm{Nb}_{2} \mathrm{O}_{5}$ structures into biologically relevant patterns. The tapping mode AFM image in Figure 1F shows spots of / surface immobilized complexes of neutravidin and single stranded DNA (ssDNA) in a bioresistant PLL- $g$-PEG background..$^{73}$ The pattern fidelity is high and there is neither remaining PMMA resist nor nonspecific adsorption of the complexes in the background. It should be possible to immobilize any DNA-tagged nano-objects to such activated surfaces as already successfully demonstrated in the micron range for phospholipidic vesicles ${ }^{72}$ and first results in the nanometer range for DNA-tagged gold colloids. ${ }^{74}$

\subsubsection{Summary of EUV-IL}

Although EUV-IL requires the use of synchrotron radiation, the resist patterns generated by this technique are of high fidelity, highly reproducible, and can cover an area as large as $3 \times 3 \mathrm{~mm}^{2}$. Compared to serial e-beam patterning, EUV-IL is much faster and can be used on insulating samples which is extremely useful if fluorescent microscopy is involved in analyzing the biopatterns, as is often the case. The combination of this advanced patterning technique and the self-assembly capability of biomolecules provides a powerful tool for the creation of biologically relevant patterned surfaces.

\subsection{Microcontact Printing}

Microcontact printing $(\mu \mathrm{CP})$ has become a very popular, alternative technique ${ }^{34,58,75}$ to pattern surfaces in the micron- and, more recently, the nanorange. ${ }^{1,76,77}$ It was originally developed by Whitesides et al. to pattern selfassembled monolayers (SAMs) of alkanethiols onto gold. ${ }^{78}$ To do so, alkanethiols were printed directly onto a gold substrate through contact with an elastomeric stamp that had been 'inked' in an alkanethiol solution. Since the stamp is made of a flexible polymer, this so-called softlithography technique can also be applied to curved and rougher substrates to produce patterns with high fidelity. ${ }^{1,2,77}$ The simplicity and low-cost of the $\mu \mathrm{CP}$ technique have made it a very attractive patterning technique for many applications. In particular, it has been adapted for printing patterns of proteins and other biomolecules onto a variety of surfaces which makes $\mu \mathrm{CP}$ interesting for an even larger scientific community. ${ }^{77,79}$

In the next paragraphs, a short introduction to $\mu \mathrm{CP}$ will be given using $\mu \mathrm{CP}$ of alkanethiols as a model system. $^{1,2,76-78}$ Further biological applications of $\mu \mathrm{CP}$, such as printing proteins,,${ }^{1,2,76-78,80,81}$ peptide sequences, ${ }^{82}$ DNA, ${ }^{83}$ or even nonfouling molecular layers ${ }^{82,84}$ to create patterns on different substrates for various applications, e.g., vesicle arrays ${ }^{80}$ will then be discussed with an emphasis on recent advances into the nanorange.

\subsubsection{Microcontact Printing: Master, Stamp, and Printing}

The production of the master, which is used for the production of stamps, is the only step in the $\mu \mathrm{CP}$ process when cleanroom facilities are needed. The master is usually produced by photolithography and reactive ion etching into silicon for micron sized features or by e-beam lithography for nano sized features. ${ }^{85-87}$ In many papers the production of the master and replica molding to produce the stamp (Figs. 2(i)-(iii)) are separately described and are not often included in the $\mu \mathrm{CP}$ process (Fig. 2(iv)-(vi)). ${ }^{1,2,75,76}$ The description of the $\mu \mathrm{CP}$ process, therefore, normally begins from the structured stamp which is then inked with a solution of alkanethiols (or certain biomolecules) and printed onto a substrate. Thus, $\mu \mathrm{CP}$ is generally referred to as a patterning technique where no cleanroom facilities are needed. ${ }^{1,2,76}$

The process of producing the stamp from the master is called replica molding (Figs. 2(i)-(iii)). ${ }^{88}$ The stamp material is in most cases poly(dimethylsiloxane) (PDMS) (Sylgard 184, Dow Corning). ${ }^{1,2}$ To form the stamp, the prepolymer of PDMS is poured over the master which, in some cases, has been treated with fluorinated silanes as a release agent, and is then cured at slightly elevated temperatures overnight. The cured PDMS stamps are demolded and, without further treatment, are ready to be inked. With standard PDMS, well defined patterns down to $500 \mathrm{~nm}$ can be created. ${ }^{89}$ To increase the long range accuracy, the PDMS precursor can also be cured on a flexible backplane. Such stamps are called 'hybrid' stamps. They combine the long range stability of the backplane with the short range flexibility of the patterned PDMS. ${ }^{90}$ 
i)

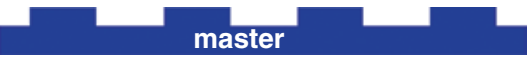

ii)

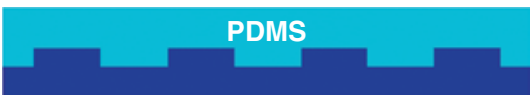

iii)

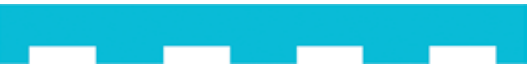

iv)

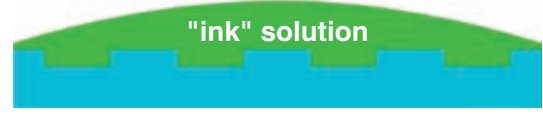

v)

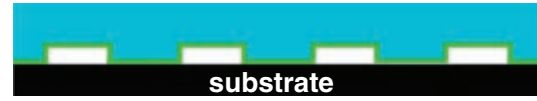

vi)

Fig. 2. Replica molding for stamp production (i)-(iii): liquid PDMS prepolymer is poured over a hard master, the polymer is cured at elevated temperatures overnight, and then released, leaving the master intact. Microcontact printing (iv)-(vi): the topographically patterned stamp is incubated in a solution of molecules (e.g., alkanethiols or proteins), the 'inked' stamp is then printed onto the substrate in the dry state, leaving a protein patterns on the surface.

Inking of the topographic stamp is done by simply incubating the stamp in the e.g., thiol solution. ${ }^{91,92}$ Depending on the concentration of the molecules, inking takes less than an hour. In the case of alkanethiol solutions, the molecules diffuse into the elastomeric stamp and form a reservoir for multiple printing steps. ${ }^{78,91}$ Alkanethiol inked stamps are dried and printed in the dry state onto gold substrates. The printing step is fast and takes less than one minute. Depending on the application the patterned substrate can be used as etching mask, ${ }^{78,93}$ or can be backfilled by dipping into another SAM solution with alkanethiols having different end groups. ${ }^{86}$ Upon making a hydrophobic/hydrophilic contrast of SAMs on a surface, the resulting pattern can be used to adsorb proteins (which adsorb preferentially to the hydrophobic areas) and then cells, which adsorb preferentially to the protein coated areas. In so doing, interesting results have been obtained from cell experiments investigating the effect of cell spreading, for example, on growth and cell cycle. ${ }^{16}$

\subsubsection{Microcontact Printing of Proteins in the Micro and Nanorange}

$\mu \mathrm{CP}$ has also been developed to print protein patterns..$^{94,95}$ The process (Fig. 2) is fairly similar to that for the alkanethiols, however, there are a few important differences. Most proteins, from aqueous solutions, stick strongly to hydrophobic surfaces ${ }^{96}$ such as the elastomeric stamp formed as described above. To print very hydrophilic proteins or peptide sequences such as poly(L-lysine), the stamp needs to be hydrophilic for better adsorption. ${ }^{82}$ PDMS stamp surfaces can be made hydrophilic through exposure to oxygen plasma for a short time. ${ }^{82}$ Due to the reactive conditions in the plasma, a hydrophilic negatively charged silica-like surface is generated. Such hydrophilic stamps should be used right after they have been prepared because the hydrophobicity of the stamp recovers due to diffusion of low molecular weight silanes from the bulk to the surface.

Again the stamp is inked by incubation in a solution of the proteins. ${ }^{88,94,95}$ The protein concentration is generally between 5 and $50 \mu \mathrm{g} / \mathrm{ml}$ and adsorption times required are approximately 40 minutes. In contrast to the short alkanethiols, proteins do not diffuse into the PDMS stamp but rather form a monolayer on the surface of the stamp. ${ }^{94}$ Thus, the stamp holds no reservoir of the 'ink' and needs to be inked again after every print. The inked stamp with the adsorbed proteins is also dried shortly before stamping. If multiple printing with a single inking step is preferred, topographically patterned hydrogels need to be used as stamp material. ${ }^{97,98}$ In Mayer's work, repetitive stamping of 100 times for a single protein type and 20 times for an array of proteins has been reported. ${ }^{98}$ The resolution, however, is then limited to ca. $50 \mu \mathrm{m}$.

Transferring the adsorbed proteins to the substrate is the most delicate step in $\mu \mathrm{CP}$ proteins. It has been reported that printing immediately after drying effects the best results concerning activity of the transferred molecules. ${ }^{88,94}$ Tan et al. showed that a prerequisite for transferring proteins in the dry state is that the substrate is more hydrophilic than the stamp..$^{99}$ The substrate can also be functionalized with a cross-linker to further increase the affinity of the proteins to the substrate. ${ }^{19}$ In any case, the adhesion of the proteins to the stamp and the substrate needs to be optimized such that the proteins will remain intact during transfer, i.e., the adhesion towards the stamp should not be stronger than the inner forces of the protein structure. Recently, Mayer et al. have presented results on printing proteins in an aqueous environment; this will be important for many of the proteins that are too fragile to be exposed to air even if only briefly in the standard $\mu \mathrm{CP}$ process. ${ }^{100}$

Printed proteins themselves present a hydrophilic surface such that further proteins can be printed on top of each other. ${ }^{88}$ Or, as in the case of Oliva, ${ }^{20}$ a stable protein can first be printed onto a surface by $\mu \mathrm{CP}$, then a second, more fragile protein such as the active extracellular component of membrane proteins, which are fused to an antibody binding fragment, can be introduced to the surface in an aqueous environment and will bind selectively to the first printed protein. Other alternative approaches to $\mu \mathrm{CP}$ include 'subtractive offset printing' and 'localized inking and offset printing' ${ }^{79}$ In 'subtractive offset printing' an unstructured flat stamp is incubated in a protein solution which homogeneously adsorbs on the PDMS. A structured master is then placed on top of the PDMS stamp, thereby removing ('subtracting') proteins in contact, such that only the protein pattern remaining on the PDMS is printed onto 


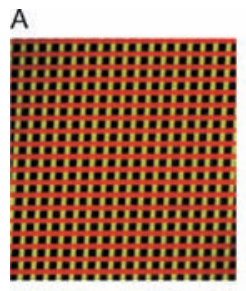

$$
\overline{50 \mu \mathrm{m}}
$$

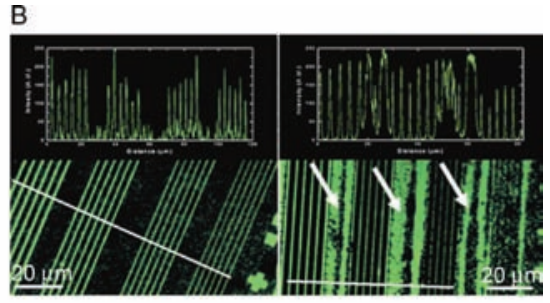

Fig. 3. Fluorescent micrographs of proteins printed by microcontact printing in the micron (A) and the nanoscale (B). (A) Image of a pattern showing three different proteins. The proteins were simultaneously printed onto the substrate. First, the flat stamp was homogeneously inked with a protein solution. A patterned silica surface was used to remove lines of proteins from the stamp by subtractive printing. The free areas could then be inked again with a different protein solution. The third protein solution was placed on the stamp accordingly. Finally the protein pattern was transferred from the flat stamp onto glass. (B) Fluorescent microscopy images of microcontact printed fluorescently labelled fibronectin lines. Comparison of a polyolefin stamp (left) with Sylgard 184 PDMS stamp (right). Both stamps show lines from $1000 \mathrm{~nm}$ down to $100 \mathrm{~nm}$. The polyolefin stamp has a higher Young's modulus compared to the standard PDMS stamp. The increase in pattern fidelity in the image from the polyolefin stamp is clearly visible. While the lines printed with the polyolefin stamp show perfect resolution down to the $100 \mathrm{~nm}$, the thinner lines printed with the standard PDMS stamp do not transfer well. Regions where the stamp has collapsed (sagging) are indicated with an arrow. (Fig 3A reprinted with permission from [88], A. Bernard et al., Adv. Mater. 12, 1067 (2000). (C) 2000, Wiley-VCH. Fig. 3B reprinted with permission from [85], G. Csucs et al., Langmuir 19, 6104 (2003). (C) 2003, Elsevier.)

the final substrate. A somewhat advanced form of 'subtractive offset printing' has been used as shown in Figure 3A to print a pattern of three different proteins at once. This was achieved by backfilling the removed proteins on the flat stamp twice with subsequent printing onto glass. For 'localized inking and offset printing', micromachined fluidic networks are used to ink a flat stamp such that only the exposed regions will transfer proteins during printing. Such microfluidic network set-ups $(\mu \mathrm{FN})$, produced either by micromachining or replica molding of PDMS, can be used for the production of heterogeneous patterns on stamps for printing as well as to direct patterning of other more fragile systems. ${ }^{79,101}$

The mechanical stability of standard PDMS (Sylgard 184) cannot support feature sizes below $500 \mathrm{~nm}$ (Fig. 3B, right) ${ }^{85,89}$ For high aspect ratios the stability is increased for stamps having a higher Young's modulus, ${ }^{102}$ thus, stamps made with a higher crosslinked PDMS ${ }^{89,95}$ or with polyolefin, as shown by Csucs et al., ${ }^{85}$ are capable of supporting nanofeatures (Fig. 3B, left). A further advantage of the polyolefin stamps is that in contrast to the low molecular weight silanes that can be left on the surface by PDMS stamps,${ }^{103-105}$ the polyolefin stamps do not leave any contamination. ${ }^{85}$ However, when using stamps with an increased modulus for higher pattern fidelity, the stamp also becomes more brittle. This can have the effect that the loss of flexibility prevents good conformal contact. Hence, high quality nanopatterns produced by $\mu \mathrm{CP}$ need to be printed on flat substrates. By using this technique, printing of protein patterns of dense lines and grids down to $100 \mathrm{~nm}$ in width has been achieved..$^{85,89,95}$ Renault et al. have also shown protein patterns printed with $100 \mathrm{~nm}$ posts and $40 \mathrm{~nm}$ grid structures (Fig. 4). ${ }^{95}$ As shown in Figure 4 the pattern fidelity is high down to lines of $100 \mathrm{~nm}$ in width for PDMS stamps with a higher Young's modulus. However, as the transferred proteins do not diffuse on the surface, ${ }^{94}$ the pattern fidelity should only depend on the stamp properties and its distortion ${ }^{106}$ as well as on the transfer yield, and may therefore be optimized.

Nanopatterns can also be produced by the novel printing technique termed supramolecular nanostamping (SuNS) that was recently introduced by $\mathrm{Yu}$ et al. ${ }^{107}$ This new method has two main differences to the stamping techniques presented so far. In addition to stamping the spatial information, chemical information can also be transferred
A
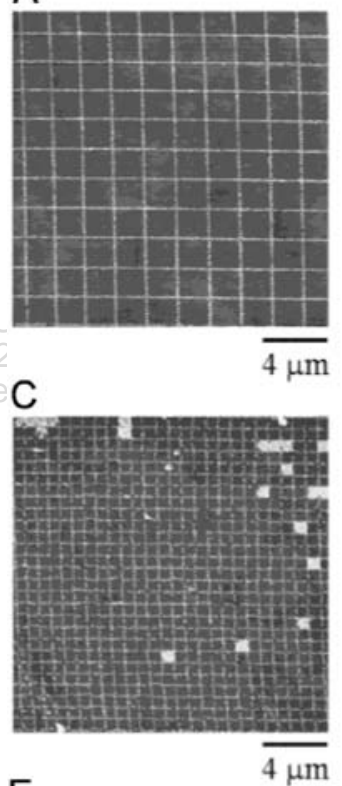

E

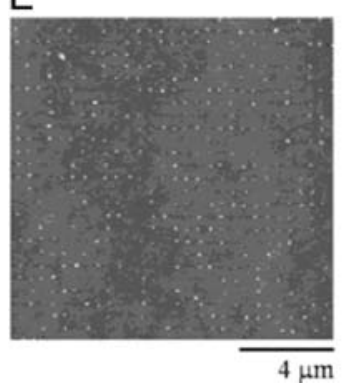

B

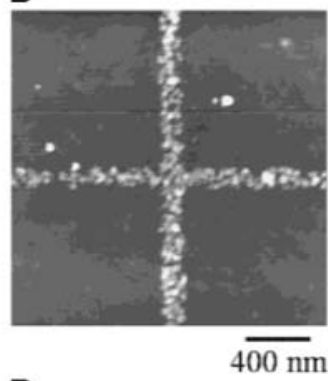

D

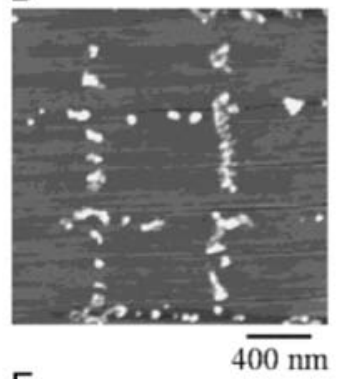

$\mathrm{F}$

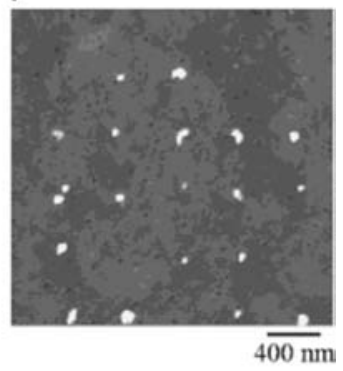

Fig. 4. AFM images of antibody grids and dots printed on glass by $\mu \mathrm{CP}$. The lines and grids presented in the images above were created with a high-resolution PDMS stamp (higher Young's modulus). Grids of $100 \mathrm{~nm}(\mathrm{~A}+\mathrm{B})$ and of $40 \mathrm{~nm}(\mathrm{C}+\mathrm{D})$ line thickness and dots of $100 \mathrm{~nm}$ $(\mathrm{E}+\mathrm{F})$ are shown. The pattern fidelity for $100 \mathrm{~nm}$ grids is very high. The $100 \mathrm{~nm}$ dots and the $40 \mathrm{~nm}$ grids still show the aimed patterns but with more disruptions. Reprinted with permission from [95], J. P. Renault et al., J. Phys. Chem. B 107, 703 (2003). (c) 2003, American Chemical Society. 
(e.g., DNA sequence). The second advantage is that the printed substrate can be used as a master. SuNS requires a master fabricated by any technique that allows for the creation of nanopatterns. In the present case, either dip-pen lithography (see also Section 3.1.1) or achromatic interference lithography ${ }^{108}$ was used to create a gold master with immobilized single stranded DNAs via a thiol linkage. The complementary thiolated DNA sequence was hybridized to such a master and a second gold substrate was brought into contact. The two substrates were put into a buffered solution kept at $80^{\circ} \mathrm{C}$ (slightly higher than the dehybridization temperature of DNA) for separation and a mirror sample of the master was obtained (Fig. 5A). The authors demonstrated the technique by creating DNA wires (Fig. 5B) and indicated that this printing method is universally applicable to all kinds of reversible molecular recognition reactions on many different substrates.

\subsubsection{Bio-Relevant Applications of $\mu \mathrm{CP}$}

$\mu \mathrm{CP}$ has been used to print patterns of hydrophobic SAMs, proteins, protein fragments, peptides, and other relevant biomolecules for a number of applications. ${ }^{82,83,86,109}$ For example, reversible hydrophobic barriers created by $\mu \mathrm{CP}$ on hydrogels has been used for generating protein microarrays. ${ }^{109}$ Functional DNA arrays have also been made by microcontact printing of the DNA directly, whereby electrostatics play the major role in molecular transfer to the substrate, to generate multiple arrays from a single loaded stamp. ${ }^{83}$ Nano- $\mu \mathrm{CP}$ should therefore enable production of functional nanoarray chips of both DNA and proteins in the near future.

$\mu \mathrm{CP}$ is also a simple and inexpensive method for the investigation of cells on protein patterns. Cell adhesive protein patterns in the size of single cells but also down to $100 \mathrm{~nm}$ have been used to investigate different cell types and their behavior including motility, adhesion, spreading

A

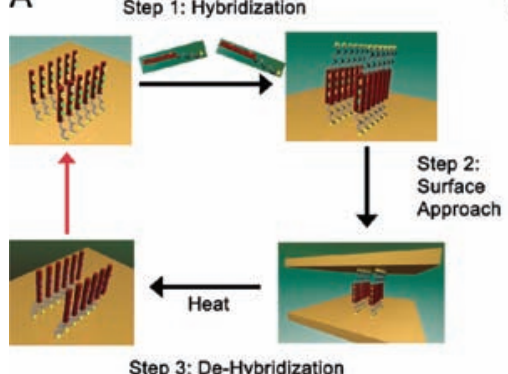

Fig. 5. Schematic of the SuNS is shown (A). After a master of nanopatterns of single stranded DNA is produced, the complementary DNA is hybridized to the surface immobilized strands (step 1). A second surface is brought into contact (step 2) and a mirror image patterned surface of the master is produced upon separation of the substrates with heat (step 3). (B) Shows AFM images of SuNS printed patterns (overview (i)), $25 \mathrm{~nm}$ (iii) and $45 \mathrm{~nm}$ (ii) thick 18 mer and 50 mer DNA wires could be printed. Reprinted with permission from [107], A. A. Yu et al., Nano Lett. 5, 1061 (2005). (C) 2005, American Chemical Society.
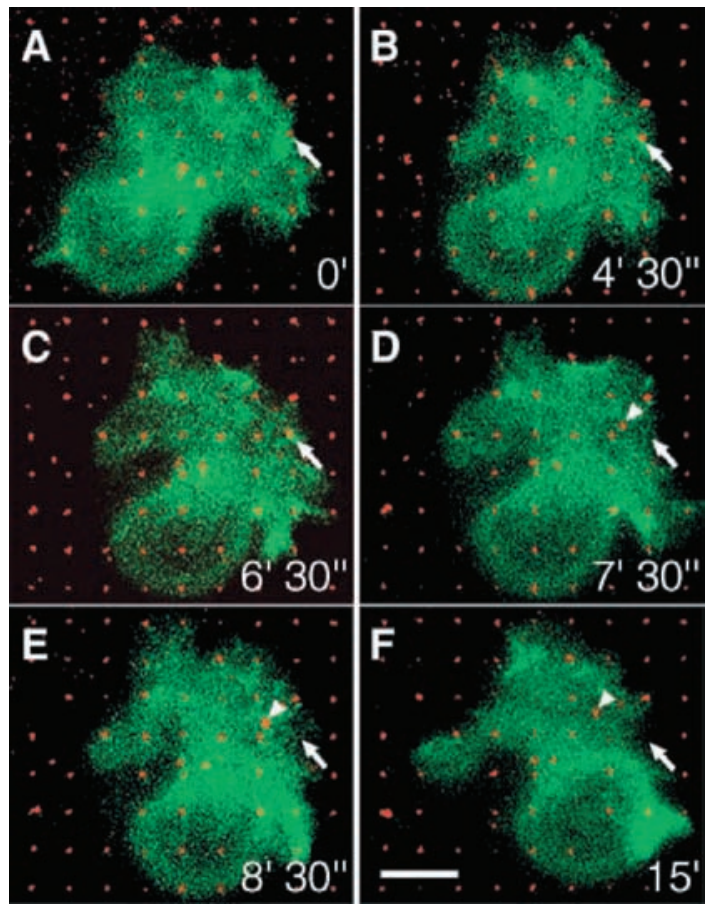

Fig. 6. Time-laps fluorescent microscopy images of B16 cells on $0.1 \mu \mathrm{m}^{2}$ fibronectin dots. The fibronectin dots were labelled red by mixing with fluorescent bovine serum albumin. The cells were visualized by $\beta 3$-integrin-GFP (green). The images show a highly motile and unspread cell. The motility of the cell has been determined using the underlying pattern. The large arrow points out a dot of the pattern which is moved by the cell (see little arrow) and internalized. The scale bar is $10 \mu \mathrm{m}$. Reprinted with permission from [86], D. Lehnert et al., J. Cell Sci. 117, 41 (2004). (C) 2004, The Company of Biologists Ltd.

and differentiation (Fig. 6). ${ }^{16,19,20,86}$ Scholl et al. and Oliva et al. have also used $\mu \mathrm{CP}$ peptide and protein patterns to guide neuron and axon growth, respectively. ${ }^{19,20} \mathrm{In}$ addition, protein patterns approaching the nanorange can be used to manipulate focal adhesion areas to differentiate cell responses due to spreading (i.e., projected footprint of cell) versus density or number of ligands (i.e., adhesive area). ${ }^{16,86}$

Furthermore, protein nanopatterns created by highresolution microcontact printing can be used as a linker to selectively direct other nanoobjects like single $100 \mathrm{~nm}$ vesicles to well-defined areas on the surface as demonstrated by Stamou et al. ${ }^{80}$ In their work, they created nanopatterns of biotinylated bovine serum albumin (BSA) by $\mu \mathrm{CP}$ and passivated the background with unfunctionalized BSA. Streptavidin was adsorbed to the surface in order to allow for the immobilization of fluorescently labeled biotinylated single lipid vesicles via the biotin/avidin linkage (Fig. 7A). The absence of co-localization of fluorescence in the fluorescent microscopy images when two populations of vesicles are used demonstrated that a high density single vesicle array could be created (Fig. 7B). This technique is a promising approach for the transportation and well-controlled immobilization of molecules incorporated into a single vesicle or in its membrane. Such an array could be useful for 


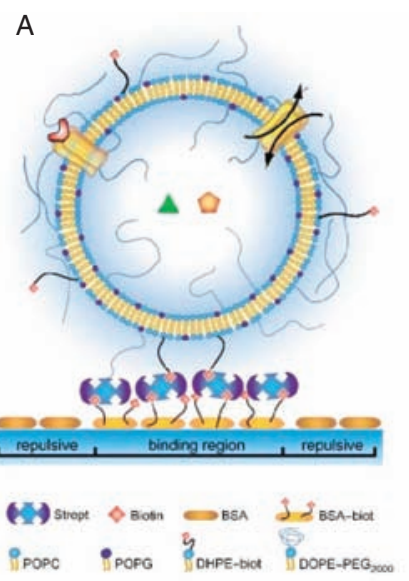

\section{B}
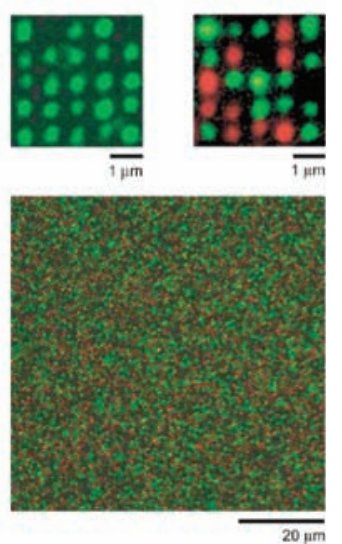

Fig. 7. (A) Single vesicle immobilization strategy. Nanospots of biotinylated BSA are created via $\mu \mathrm{CP}$. The background is passivated with BSA. Streptavidin is then used to immobilize biotinylated vesicles onto such surfaces. (B) A fluorescent microscopy image of a single vesicle array consisting of one (left) or two (right) vesicle populations is shown. A complex high-density vesicle array could be created (bottom). Reprinted with permission from [80], D. Stamou et al., Angew. Chem.-Int. Ed. 42, 5580 (2003). (C) 2003, Wiley-VCH.

simultaneous screening (drug candidates) especially if the approach is extended to native vesicles.

\subsubsection{Summary of $\mu \mathrm{CP}$}

$\mu \mathrm{CP}$ has been applied successfully not only to pattern alkanethiols SAMs, which it has been developed for, but also to many other 'ink' molecules including proteins, peptides, and DNA. It is a parallel technique which allows high fidelity patterns to be created from the centimeter to the low nanometer range. The low cost of microcontact printed patterns and the ability to pattern biomolecules also make it a very attractive tool for bioapplications.

\subsection{Nanoimprint Lithography (NIL)}

In 1995 Cou et al., ${ }^{37,110}$ proposed nanoimprint lithography (NIL) based on a fundamentally different principle from that for conventional lithography. NIL has the potential to fulfill the need for a low-cost and high-throughput nanopatterning technology which could be applicable not only in microelectronics but also in other fields such as biology, medicine, or information storage. This alternative lithography method consists of two steps: compression molding (imprint) and pattern transfer. During the imprint step, a nanostructured rigid mold is pressed into a thin resist layer spin coated onto a substrate (Fig. 8A(i)). The resist (e.g., PMMA) is deformed by the mold when heated above its glass transition temperature (Fig. 8A(ii)). Due to this heating cycle the method is also termed 'hot embossing'. The mold is removed when the resist is cooled below its glass transition temperature (Fig. 8A(iii)). A reactive ion etching (RIE) step is used to remove the residual resist in the compressed areas (Fig. 8A(iv)).

For reproducible fabrication of high-resolution nanopatterns using NIL, many factors, such as the viscoelastic properties of the polymer (e.g., for complete filling of the mold structure) and the demolding behavior (antisticking layer, fluorinated molds ${ }^{111}$ ), must be considered. In the following, a general overview of the technique will be presented with a focus on NIL based applications in biology. For more details about the manufacturing process please refer to Refs. [112-114].

\subsubsection{NIL Process}

Since NIL is not based on a chemical modification of a resist caused by light radiation, the resolution is not limited by factors known to conventional lithography such as wave diffraction, scattering, and interference, rather, it is limited by the template fabrication process. The stamp fabrication, probably not only the most critical but also the most time consuming step, can be done by $e$-beam lithography ${ }^{87}$ or by EUV-interference lithography ${ }^{115}$ (for details about EUV-IL see also Section 2.1.1). In addition to the fabrication, the long term stability of the mold is another critical issue. The heating cycle of the NIL process causes wear

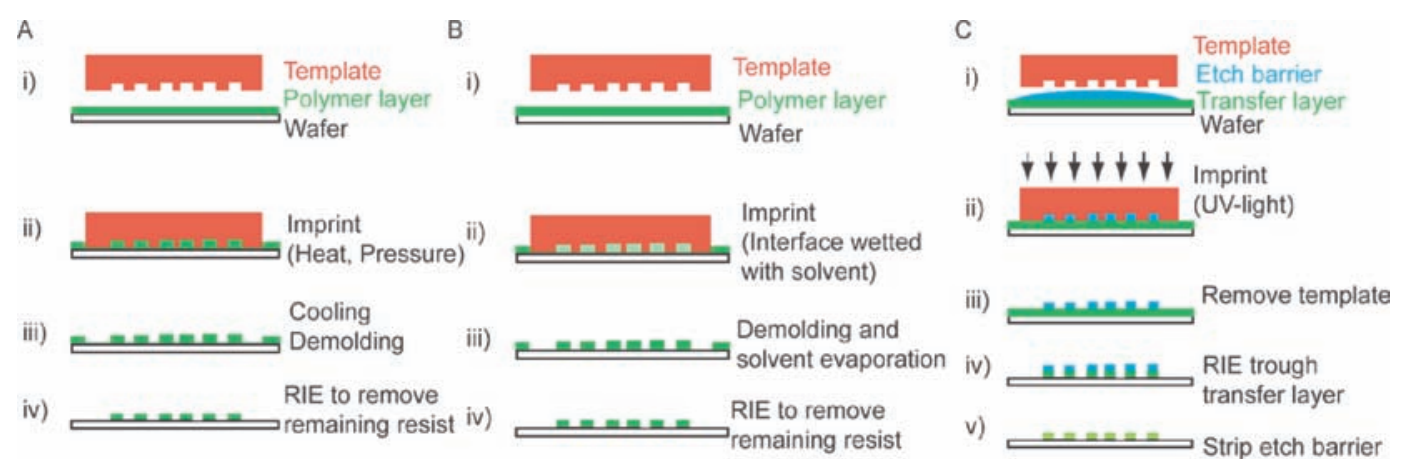

Fig. 8. Schematic of the Nanoimprint Lithography (NIL, 'hot embossing') (A), Solvent-Assisted Micromolding (SAMIM) (B), and Step and Flash Imprint Lithography (S-FIL) (C). (A) NIL allows for the creation of nanopatterns via the imprint of a template into a polymer layer by using heat and pressure (i+ii). After cooling and demolding (iii), the patterns are transferred via RIE (iv). ${ }^{37}$ The basic principle (imprint and patterns transfer via RIE) stays the same for SAMIM and S-FIL but heat and pressure are avoided. (B) SAMIM uses a solvent to wet the interface while imprinting (ii). ${ }^{116}$ (C) S-FIL needs an additional layer (i) which is hardened upon exposure to UV-light (ii). ${ }^{17}$ 
and stress to the stamp. This aging of the stamp can affect the pattern fidelity.

Three closely related methods of NIL were developed to overcome this aging problem of the stamp: SolventAssisted Microcontact Molding ${ }^{116}$ (Fig. 8B, SAMIM), Step and Flash Imprint Lithography ${ }^{117}$ (Fig. 8C, S-FIL), and plasticizer-assisted imprinting. ${ }^{118}$ Solvent-Assisted Microcontact Molding (SAMIM) creates patterns in a polymer by using a soft stamp (in general PDMS) and an appropriate solvent ${ }^{119}$ which wets the interface during the imprint process. The topography created upon evaporation of the solvent is a negative replica of the relief pattern on the stamp. For more details see e.g., Gates et al. ${ }^{34}$ and the references therein. In S-FIL a low viscosity photocurable solution (etch barrier, containing low molecular weight monomer and photoinitiator), in addition to the spin-coated polymer layer (transfer layer), is used. As a substitute of pressure and heat, UV light is used in the imprint step to photopolymerize the etch barrier. The use of a transparent quartz template makes it possible to align the template with respect to existing features on the substrate. The absence of a heating step further facilitates the alignment procedure as potential problems due to thermal expansion are avoided. This is a basic requirement if several lithography steps are needed, e.g., in the creation of integrated circuits. For more details about S-FIL please refer to a review by Resnick et al, ${ }^{120}$ Plasticizer-assisted imprinting allows for the creation of nanopatterns at room temperature with low pressure in nonthermoplastic polymers or biomaterials by the addition of plasticizer.

Using NIL, it is possible to pattern entire $4 "$ wafers in one step as it is a parallel process. ${ }^{121}$ The size of the imprinted area can further be increased by using step and stamp imprint lithography which allows for the repeated imprint of the same mask on the same substrate. ${ }^{122}$ Alternatively, roller nanoimprinting is a possibility which heats the resist over its glass temperature locally, while rolling, to imprint. ${ }^{123}$

\subsubsection{Bio-Relevant Application of NIL}

The achieved polymer structure by NIL can be used in different ways. On the one hand, the nanostructure can serve as device itself assuming that the polymer has functionality (e.g., optical properties ${ }^{124}$ ). On the other hand, a variety of bio-applications are also possible by incorporating the polymer contrast into other devices. For example, a nanofluidic device for DNA stretching can be created if the mold is replaced with a channel template and the demolding is omitted. ${ }^{125}$ In addition, the nanostructured polymer can be used to guide molecular motors (guidance of actin filament sliding); such controlled movement of biomolecules is a basic requirement for 'lab-on-chip' applications. ${ }^{126}$ Another challenge is to introduce nanopillars in high density inside of microchannels. These arrays could serve as an artificial gel in integrated capillary electrophoresis chips. Imprint lithography is a low-cost technology which can create such arrays either on a $\mathrm{SiO}_{2} / \mathrm{Si}$ substrate including an etching step or by direct hot embossing into a plastic device. ${ }^{127}$ NIL patterned surfaces are also helpful to address questions towards the importance of nanostructures (topography or chemistry) to cellsurface interactions. ${ }^{128-130}$

There are also different ways to transfer the (polymer) structure created by NIL into biologically relevant patterns. It is possible, for example, to combine NIL and anodization of aluminum, as demonstrated by Matsumoto et al., ${ }^{131}$ for the creation of a high density nanopattern of DNA. A $\mathrm{SiC}$ mold is imprinted in aluminum and upon anodization an ordered pore structure is achieved. The pores can be filled with gold and thiolated fluorescently labeled single stranded DNA can be surface immobilized and observed with the fluorescent microscope.

Additionally, the polymer structure can be used in two different ways: (a) as dry etching mask ${ }^{132,133}$ or (b) as a step in a lift-off process as described by Falconnet et al. ${ }^{134}$ Hoff et al. were able to use nanoimprinting to generate high-resolution protein nanopatterns. In a first step, spin coated PMMA is patterned by NIL (Fig. 9A). RIE was then used to remove PMMA residues and to passivate the $\mathrm{SiO}_{2}$ with a thin layer of $\mathrm{CF}_{x}$ polymer created during the process. The remaining PMMA resist was stripped off and a covalently bound monolayer of aminosilane was adsorbed onto the $\mathrm{SiO}_{2}$ by vapor deposition. Biotinsuccinimidyl ester was then covalently bound to the silane layer. Such a surface, when exposed to a streptavidin containing solution, can be used to selectively immobilize any kind of biotinylated target protein. $75 \mathrm{~nm}$ line patterns of biotinylated BSA, visualized in the fluorescent microscope by the addition of rhodamine labeled streptavidin, could be achieved with this approach (Fig. 9B).

Another approach to create biologically relevant patterns in the nanometer range is the combination of the NIL technique with the Molecular Assembly Patterning by Lift-off (MAPL) process, described in Section 2.1.1. ${ }^{134}$ If the oxide surface is pre-patterned by NIL instead of the standard photolithography technique biologically relevant PLL- $g$-PEG/PEG-biotin nanolines in a $\mathrm{Nb}_{2} \mathrm{O}_{5}$ background could be created. ${ }^{134}$

\subsubsection{Summary of NIL}

NIL is a promising low-cost approach which was successfully applied to different biologically related questions. The ability to produce a large number of identical nanostructured samples opens up the possibility to create nanoarrays for biosensing. Additionally it is a great advantage since biological studies (cell experiments) require many experiments for meaningful statistics. Currently there are still production problems (e.g., alignment, demolding, fouling of the mold) to be solved in order to further increase the pattern fidelity especially if a dense structure 
A

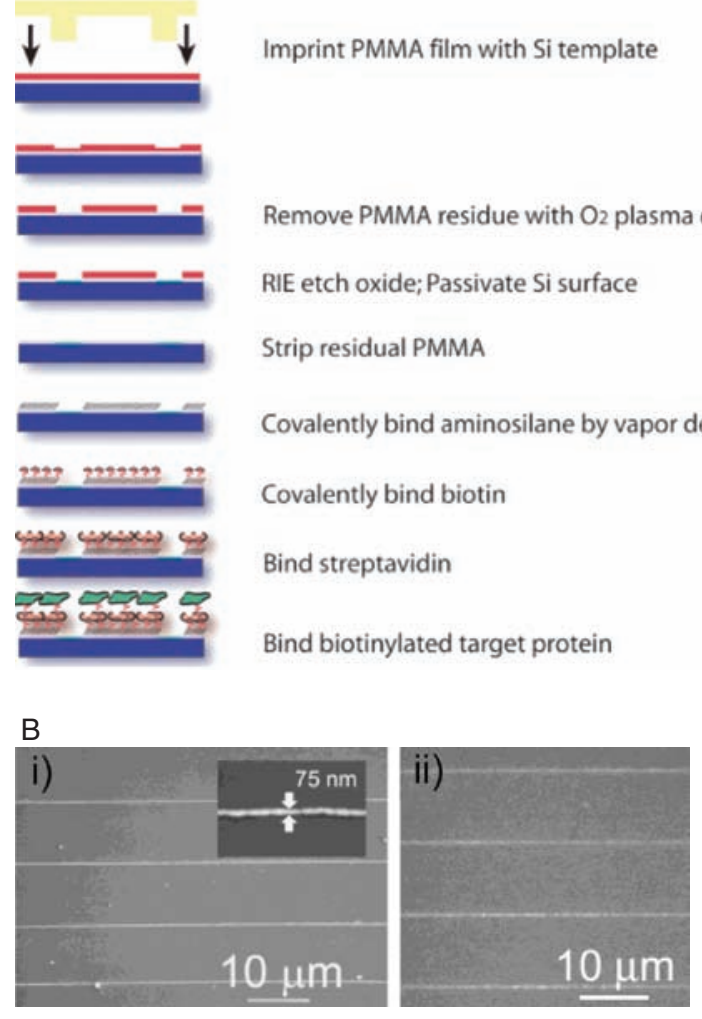

Fig. 9. (A) A patterning method based on NIL for the creation of biologically relevant nanopatterns is shown. First, a resist is patterned by NIL. Then, reactive ion etching removes PMMA residues and passivates the exposed $\mathrm{SiO}_{2}$ areas. After stripping off the remaining PMMA, an aminosilane monolayer is deposited in the non-passivated $\mathrm{SiO}_{2}$ areas. Biotin-succinimidyl ester is then linked to the aminosilane layer. Finally, streptavidin is used to selectively immobilize biotinylated target protein (biotinylated BSA in the presented case). (B) SEM image of oxide nanolines on $\mathrm{Si}$ (i) and fluorescence micrograph of nanoline patterns of biotinylated BSA and bound rhodamine labeled streptavidin (ii). Reprinted with permission from [132], J. D. Hoff et al., Nano Lett. 4, 853 (2004). (C) 2004, American Chemical Society.

over a large area is needed. However, NIL may eventually enable the immobilization of single biomolecules onto a surface in well-defined areas. Nanofluidic devices created by NIL related methods may further allow for the manipulation of biomolecules, which would be particularly useful in the creation of 'lab-on-a-chips'.

\subsection{Colloid Lithography}

Colloidal particles have attracted large interest in surface engineering due to a number of advantageous properties. Nanosized particles not only exhibit interesting physical properties originating from their size ${ }^{135-137}$ they have also become widely commercially available. Metal, metal oxide or polymer (latex) particles can be produced in almost all sizes and with narrow size distributions ([138] and references therein). Furthermore, scientists are now developing methods to immobilize nanoparticles on substrates as monolayers, ${ }^{139-142}$ photonic crystals, ${ }^{143-147}$ patterned particle arrays, ${ }^{148-153}$ or as single particles, ${ }^{154-157}$ thereby opening up the possibilities for applications of particle-modified surfaces in many research areas.

In this section, methodologies and concepts using nanosized colloidal particles with potential applications in the broad field of nanobiotechnology will be discussed. In general, the ways in which nanoparticles may impact biologically relevant nanopattern formation fall into one of three categories. First, nanoparticles can be used to produce nanopatterns with a biological function on substrate surfaces, either directly through the adsorption of single (functionalized) particles, patches of particles, or monolayers of particles, or indirectly by acting as a mask as in colloidal lithography. ${ }^{38,139,140,158-165}$ This latter use of nanoparticles presents one of the most promising ways by which nanoparticles can be used to pattern biologically useful nanopatterns. Second, colloidal nanoparticles may be used as a platform for the adsorption of biomolecules for use in biosensing devices or as markers. ${ }^{152,163-169}$ The role of the particle, in this case, may be two-fold: nanoparticles can be used as an integral part of such a biosensing device, e.g., providing conductivity by using conducting particles, ${ }^{166,170-172}$ or by incorporating particles into a system that have a target biomolecule (pre-) adsorbed to its surface. ${ }^{173}$ Third, colloids functionalized with biomolecules (e.g., DNA) can be used to attach particles at specific locations on a pattern using biological recognition systems, e.g., assembling (gold)colloids on surfaces using biological molecules. Although this is a promising bottom-up approach for fabrication of gold nanowires and devices, this third application falls into the realm of bionanotechnology, i.e., using biology to create nanostructures, and as such is beyond the scope of this review.

\subsubsection{Nanostructures Created by Nanoparticles: Nanosphere Lithography (NSL)}

One of the most common ways of producing nanopatterns using particles was introduced in the early eighties by Fischer et al. ${ }^{158}$ and soon after extended by Deckman et al. ${ }^{139}$ Initially termed 'natural lithography', particle monolayers were first used as masks for contact imaging ${ }^{158}$ and later used as masks for etching and material deposition. ${ }^{139}$ The basic idea of this process is straightforward. First, a monolayer of particles is formed on a substrate. In a second step, a material is deposited on top of the particle layer. The particle layer thereby acts as a mask such that material is only deposited in the empty spaces of the hexagonal particle assembly. After lift-off of the particles, hexagonal nanostructures of deposited material then remain on the surface (see Fig. 10A). At a later stage, Hulteen et al. developed double layer masks over relatively large areas $(10-100 \mu \mathrm{m})$ (see Fig. 10A(ii)) to produce nanoparticle arrays, as characterized by AFM. ${ }^{140}$ 


\section{A}
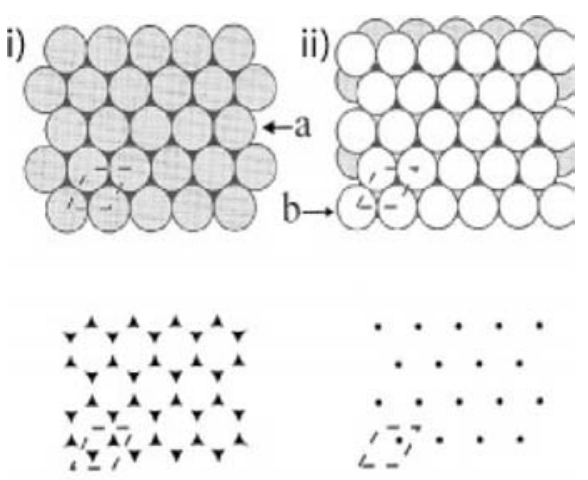

-,$\cdot$
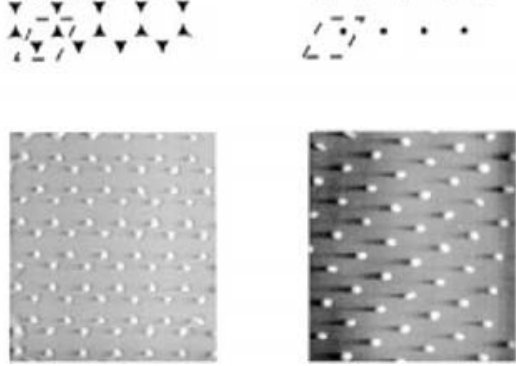

B
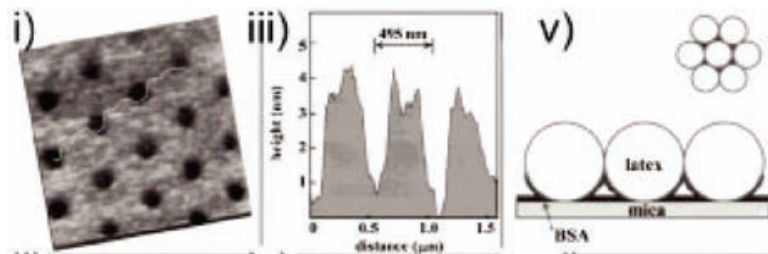

ii)

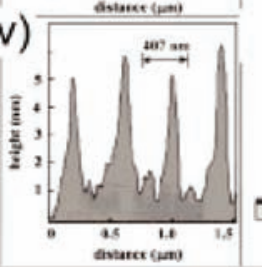

vi)

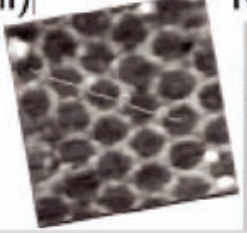

astarce 6 an

Fig. 10. (A) Nanosphere lithography of single and double layer masks. Top left (i): schematic representation of a single layer mask, middle (i): periodic particle array with a unit cell (dashed line) resulting from depositing material on the mask and removing the particles and bottom (i): AFM image $\left(1.7 \times 1.7 \mu \mathrm{m}^{2}\right)$ of $\mathrm{Ag}$ deposited on mica using $250 \mathrm{~nm}$ latex spheres. (ii) same as (i) but for double layer masks. Reprinted with permission from [140], J. C. Hulteen and R. P. Van Duyne, J. Vac. Sci. Technol. A: Vacuum, Surfaces, and Films 13, 1553 (1995). (C) 1995, American Institute of Physics. (B) Nanosphere lithography used to directly pattern protein structures by co-adsorbing bovine serum albumin (BSA) with latex particles. At high BSA/latex colloid ratios, structures as observed in (i) are obtained, (iii) shows a height profile from (i), (v) is a schematic representation of the latex particles co-adsorbing with BSA at high BSA/latex ratios, while reducing the BSA/latex ratio leads to less densely packed structures as observed in (ii, iv, vi). Reprinted with permission from [162], J. C. Garno et al., Langmuir 18, 8186 (2002). (C) 2002, American Chemical Society.

In recent years, a variety of systems have been studied and the technique has attracted increasing interest due to its advantages: it is an inexpensive, inherently parallel, high-throughput nanofabrication technique. For an interesting feature article on advances in particle or nanosphere lithography, refer to Heynes et al. ${ }^{137}$ Despite these advances, the difficulty to produce defect-free particle monolayers remains and this in turn restricts the large-scale production of nanosphere lithographic patterns and limits the number of substrate materials that can be used to, primarily, glass substrates and silicon wafers.

Several ways of producing particle monolayers and the study of the formation processes that lead to such 2D crystals have been reported, including spin-coating, ${ }^{136,140}$ drop drying, ${ }^{17-176}$ and evaporation of the solvent using a Peltier element. ${ }^{141,177}$ Wang et al. used a slightly more complicated way of producing their monolayers, which yields monolayers of comparable quality. ${ }^{142}$ Ormonde et al. have used convective self-assembly of the nanospheres to increase the pattern fidelity of the particle mask compared to drop coating of the particles. ${ }^{178}$

However, it remains the case that the number of achievable patterns, by NSL, is limited and is defined by the particle monolayer and the interparticle holes formed in that layer. In recent years, progress has been made to decrease the size of these features not only by using smaller particles but also by tilting the substrate with the particle monolayer relative to the deposition direction. This significantly decreases the interparticle spacing which can be patterned. ${ }^{179}$ Alternatively, Snoeks et al. used an ion-beam to deform the particle monolayers such that the interparticle spacing was reduced (by 'squeezing' the particle layer together) and consequently smaller nanopatterns could be produced, ${ }^{161}$ while Sun et al. used a laser to locally change the morphology of the resulting nanopatterns effectively. ${ }^{136}$

An alternative approach to 'classic' nanosphere lithography was presented by Michel et al. to produce biologically relevant nanostructures. ${ }^{159}$ They adsorbed colloidal latex particles (100 $\mathrm{nm}$ diameter) on a titanium oxide surface to form a nanopattern of randomly adsorbed colloidal particles (see Fig. 11). Note that in this case the particles are interacting electrostatically with the surface, and as such form arrays of randomly adsorbed, separated particles. This is in contrast to standard nanosphere lithography, in which interactions between the colloidal particles and the substrate are minimized such that capillary forces drag the particles together during the drying process to form hexagonal colloidal crystals. In this alternative approach, the adsorbed particles were used as a mask in a subsequent ion-beam etching process, ${ }^{38,160}$ such that the titanium oxide surface (only $12 \mathrm{~nm}$ thin) was etched away, except where protected by a particle, revealing the underlying $\mathrm{SiO}_{2}$ substrate. In such a way, a $\mathrm{TiO}_{2} / \mathrm{SiO}_{2}$ nanostructure was created (Fig. 11).

Further functionalization of this metal oxide nanostructure was achieved using a process called Selective Molecular Assembly Patterning (SMAP) ${ }^{61}$ where an alkanephosphate $\mathrm{SAM}$ was specifically adsorbed onto the $\mathrm{TiO}_{2}$ pillars and the PLL- $g$-PEG was adsorbed on the $\mathrm{SiO}_{2}$ background. Such a surface will be resistant to protein adsorption in the silica background, due to the PLL- $g$-PEG, and proteins will readily adsorb to hydrophobic SAM on the titania nanopillars. In Figure 11E(i) such a nanostructured surface is imaged using AFM and in Figure 11E(ii) 

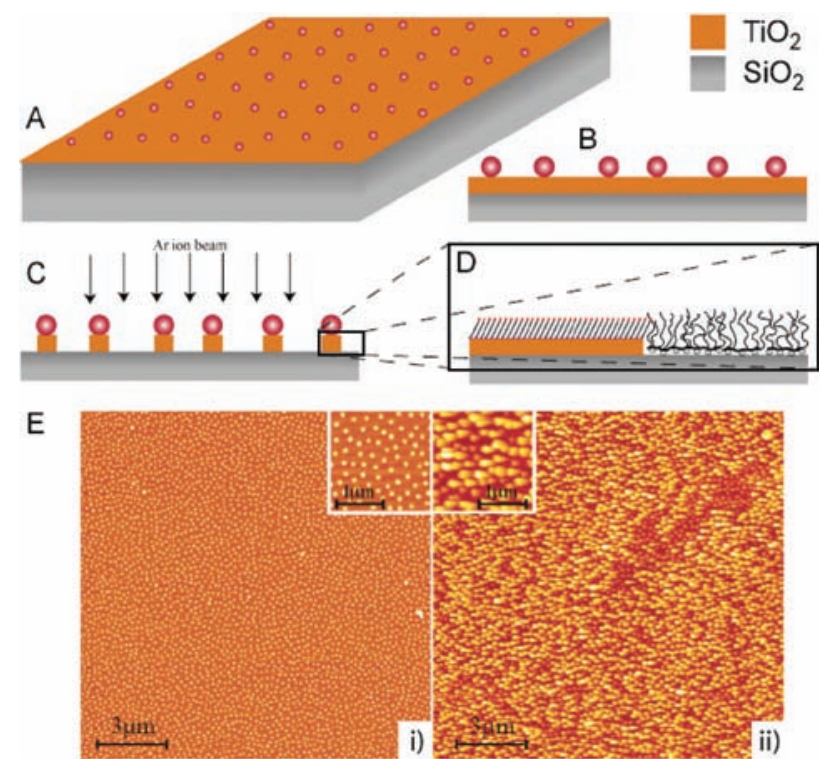

Fig. 11. Schematic representation of colloid lithography combined with selective molecular assembly patterning (A)-(D). (A) Random adsorption of nano particles on a silicon wafer coated with $12 \mathrm{~nm} \mathrm{TiO}$. (B) Side view. (C) Argon ion-beam etching removes the titanium oxide only where it is not protected by colloidal particles. (D) After removal of the latex particles, the $\mathrm{TiO}_{2}$ areas where modified using an alkane phosphate SAM that specifically adsorbs to the $\mathrm{TiO}_{2}$ leaving the silica surface uncovered. In a next step, PLL- $g$-PEG (see text) is adsorbed on the silica background rendering the background protein resistant. (E) AFM images of such a surface (i). On the right side (ii), biotinylated vesicles were adsorbed to the titania pillars by first adsorbing streptavidin (the background is resistant to streptavidin adsorption). Reprinted with permission from [159], R. Michel et al., Langmuir 18, 8580 (2002). (C) 2002, American Chemical Society.

biotinylated vesicles were immobilized onto the pattern via a biotin-streptavidin linkage.

\subsubsection{Applications of Nanoparticles and Nanoparticle Nanopatterns}

As mentioned earlier, nanosphere lithography is of special interest for biosensing applications due to the fact that the resulting pattern is again a pattern of nanoparticles (see Fig. 10A). This is of interest since nanoparticles are often characterized by interesting physical properties, be they optical, ${ }^{163,165,180,181}$ magnetic, ${ }^{182}$ or electronic, ${ }^{183}$ that change upon adsorption of biomolecules, such that they can also be used to read-out signals in biosensing devices.

Haes et al. and Frederix et al. used exactly this approach to explore the potential of using a nanosphere patterned surface for biosensors. ${ }^{163-165,184}$ In this case, polystyrene beads were used to produce the colloidal monolayers and subsequent deposition of $\mathrm{Ag}$ led to colloid nanostructures as observed in Figure 12. There, the influence of a thermal annealing step on the Ag nanopattern can be observed. ${ }^{165}$ The effect that they take advantage of for their biosensor is termed localized surface plasmon resonance (LSPR), an effect that originates from the extraordinary

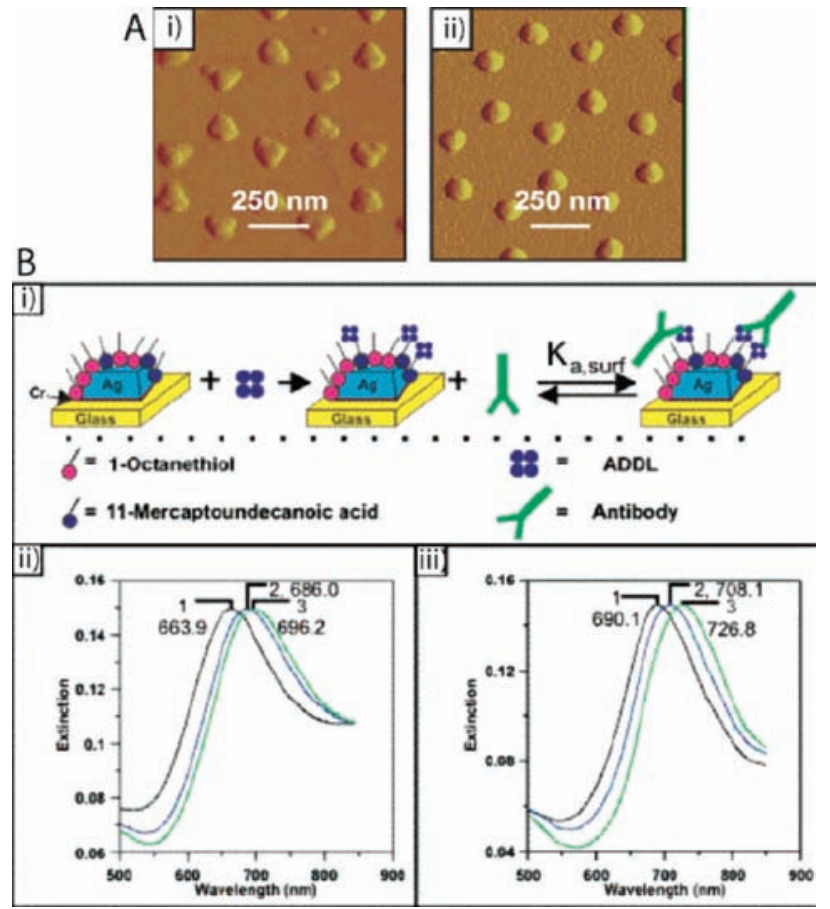

Fig. 12. (A) Tapping-mode AFM images of typical nanosphere lithography patterns of $\mathrm{Ag}$ on glass after solvent annealing (i) and after thermal annealing at $650{ }^{\circ} \mathrm{C}$ (ii). Reprinted with permission from [165], A. J. Haes et al., J. Phys. Chem. B 108, 6961 (2004). (C) 2004, American Chemical Society. (B) Schematic representation of a Ag nanodot biosensor (i). Ag nanodots are functionalized with a mixed SAM, then ADDL (a peptide thought to be playing a key role in Alzheimer disease) is immobilized on the SAM and finally, an anti-ADDL antibody is attached to the ADDL on the nanobiosensor. LSPR spectra of all steps mentioned in (i) at small concentrations of anti-ADDL (ii). The same spectra are shown, except at much higher concentrations of anti-ADDL and therefore show a larger increase of the extinction maxima (iii). Reprinted with permission from [164], A. J. Haes et al., Nano Lett. 4, 1029 (2004). (C) 2004, American Chemical Society.

optical properties of nobel metal nanoparticles. The LSPR refers to the ability of e.g., Ag nanoparticles to oscillate their conduction electrons collectively, which induces strongly enhanced electromagnetic fields surrounding the nanoparticle and will, in turn, determine the sensing volume in which sensing can occur. ${ }^{164}$ This LSPR effect, not present in bulk $\mathrm{Ag}$ or $\mathrm{Au},{ }^{185}$ will also affect resonant Rayleigh scattering, with an efficiency equivalent to that of $10^{6}$ fluorophores, and absorption with extremely large molar extinction coefficients. ${ }^{165}$ It is this extinction, which is extremely sensitive to molecules adsorbed onto the surface of the particles, that can be measured and enables the transduction of chemical binding events into optical signals (for details see Ref. [165]). Thus, Ag nanodots prepared by nanosphere lithography act as effective chemical and biological optical nanosensors. By simply monitoring changes in the UV/Vis absorption band of the nanoparticles, adsorption of chemical or biological species could be detected. A modification of this technology uses nanometer size holes in a conductive gold film, instead 
of the particles, for the generation of LSPR. This has the advantage that it can be readily combined with other conventional biosensing techniques such as QCM-D, SPR, or electrochemical sensing. ${ }^{47}$

These very sensitive biosensors usually function by first adsorbing a mixed SAM monolayer onto the Ag nanodots. Then, a protein of interest is adsorbed to that molecule and as a last step an antibody that interacts with the protein is introduced. Each of these steps is observable by UV/Vis spectroscopy due to a slight change in the extinction maxima of the LSPR spectra (see Fig. 12 for an example system). ${ }^{163}$ As a first practical system, a biosensor based on this working principle, for the possible detection of Alzheimer disease, was developed and tested. ${ }^{164}$ In Figure 12, a schematic representation of this sensor is given, thereby presenting a nice example of how colloidal nanoarrays might be useful in the future as biosensing devices due to their size-dependent, favorable properties.

In addition, the use of nanoparticles might significantly enhance the properties of an existing biosensing device by increasing the surface area available for capturing a target molecule as demonstrated recently. ${ }^{152,167}$ In our own work, we have recently presented an approach to increase the sensitivity of current biosensing devices (based on optical waveguide techniques) through the use of nanoparticles. Guided self-assembly of $80 \mathrm{~nm}$ silica particles was used to direct the particles to given areas that later act as sensing spots. Chemical functionalization of these particles and the surface leads to an inherently protein resistant system of patterned nanoparticles in a protein resistant background. Only adsorption of desired molecules to the linking sites on the particles (in our case biotin-streptavidin) is possible. Such particle arrays with only specific interactions may be used to increase the signal in standard biosensing devices. ${ }^{152}$

Nanoparticles have also contributed to biosensing and biovisualization as markers. For example, nanoparticles have enabled detection of single molecule interactions by visualizing individual gold colloid labels optically ${ }^{168}$ or by means of scanning force microscopy. ${ }^{169}$ In both cases, nanoparticles functionalized with antibodies ${ }^{168}$ or DNA ${ }^{169}$ have been used to later image these particles after they have been bound to the protein and complementary DNA, respectively.

Colloidal lithography as presented in the above examples can be used as an efficient parallel way of producing various nanostructures. However, in addition to the use of colloidal layers as a mask for etching and material deposition, one can also think of completely covering the nanoparticles and thereby creating surfaces of nano 'bumps'. Such surfaces were produced and used in cell experiments to examine the reaction of cells to nanostructured surfaces. ${ }^{186}$ Garno et al. also used colloidal monolayers in a similar way to directly pattern protein nanostructures on various substrates. ${ }^{162}$ A mixture of BSA (or IgG) and latex particles was prepared and adsorbed on a substrate. After dissolution of the latex particles, hexagonal protein nanostructures could be observed on the surface covering the free areas between the particles (see Fig. 10B for details).

\subsubsection{Summary of NSL}

To conclude, (nano)particles will likely play an important role in various areas of nanobiotechnological applications. Today, they appear to have the biggest impact in the field of biosensing where their unique properties as discussed above can shine the most. Tremendous progress has been made in arranging particles on surfaces (in almost every imaginable way), but generating large-scale particle assemblies that are biologically useful while preventing unspecific adsorption of biological macromolecules remains a challenge for the production of future applications. As a general trend, we see ways to very precisely position particles (single particles, few particles, particle assemblies) on surfaces but these often lack the needed surface functionalization properties for the use in nanobiotechnological applications. On the other hand, when particle (and substrate) do have the properties needed for such applications, arranging these particles becomes less trivial. It will be the combination of these two aspects, i.e., precise control of particle positioning and tunable, biologically relevant particle and substrate functionalization, that will open the path to further nanoparticle applications in nanobiotechnology.

\subsection{Block-Copolymer Micelle Lithography}

\subsubsection{Block-Copolymer Micelle Lithography Nanopatterns}

Block-copolymer micelle lithography is a so called bottom-up approach to nanopattern surfaces and it takes advantage of the self assembly behavior of blockcopolymers. These are used as building blocks to form uniform monomicellar films and finally to produce a nanometer-sized pattern on a flat substrate..$^{39,187-190}$ In an appropriate solvent, amphiphilic diblock copolymers form micelles where the more soluble block forms a shell around the less soluble block. ${ }^{191}$ A metal precursor salt is added into the polymer solution and reduced inside the core of the micelle forming a nanoparticle. The range of materials to be used as precursors is vast, it includes both noble metals and various oxides. ${ }^{192}$ Through oxygen plasma treatment, the polymer is removed and nanometer sized dots cover the sample surface uniformly in a hexagonal pattern. Figure 13A visualizes the principle of blockcopolymer micelle lithography. Figure 13A(i) depicts the formation of an extended monomicellar film and the following plasma treatment results in a uniform distribution of nanoparticles. Combining this technique with photo or e-beam lithography enables the deposition of nanodots in two different length scales in periodic or even 


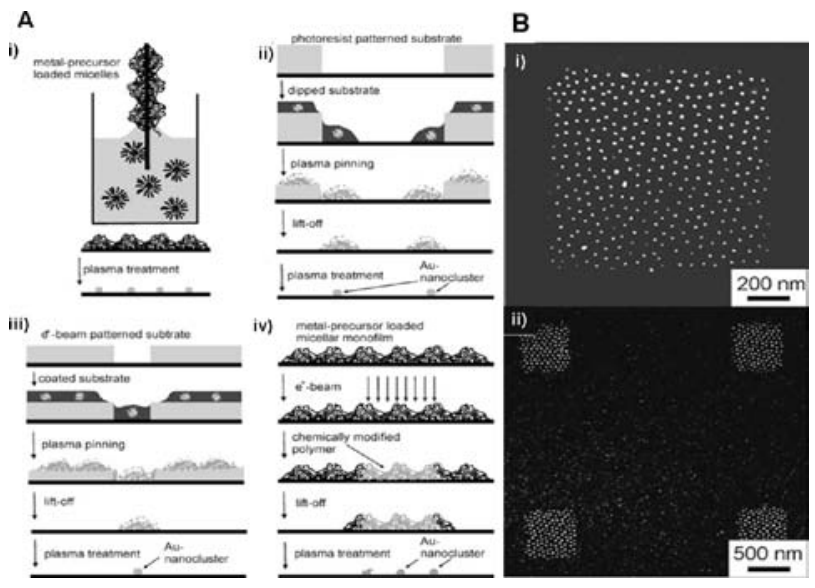

Fig. 13. (A) Different principles of block copolymer micelle lithography: Formation of a monomicellar film and ensuing plasma treatment (i), photolithography (ii), and e-beam lithography (iii) are then used to produce a prestructured surface and the monomicellar film is used as a negative e-beam resist (iv). (B) SEM images of $7 \mathrm{~nm}$ large Au-nanodots after e-beam lithography, lift off and plasma treatment: the distance between the dots is about $73 \mathrm{~nm}$ (i), squares of nanodots separated by $2 \mu \mathrm{m}$ (ii). Reprinted with permission from [197], R. Glass et al., Nanotechnology 14, 1153 (2003). (C) 2003, Institute of Physics Publishing.

aperiodic patterns. ${ }^{193,194}$ Figures $13 \mathrm{~A}(\mathrm{ii})$ and (iii) shows a prestructured surface fabricated by photolithography and, by e-beam lithography, respectively. The written areas are removed after developing, which results in a prestructured resist film. After exposure to the polymer solution, plasma treatment and lift-off, the nanoparticles are arranged on the surface according to the original prepattern in the resist. In Figure 13A(iv) the monomicellar film itself is used as a negative e-beam resist. This allows for the fabrication of structures as small as a few nanometers because the resolution is not limited by the radius of gyration of the resist material, as it is in standard e-beam lithography. ${ }^{195}$ Figure 13B shows the SEM image of different patterns of $\mathrm{Au}$ nanodots after e-beam lithography. The e-beam exposed area was reduced from $1 \times 1 \mu \mathrm{m}^{2}$ (i) down to $500 \times 500 \mathrm{~nm}^{2}$ (ii).

The self-assembled pattern depends on molecular parameters which can be controlled and tuned. The distance between the dots depends on the length of the polymer blocks, while the size of the nanoparticles can be influenced by the concentration of precursor salt. Spatz et al. ${ }^{196}$ produced nanodots with a size between 1 and $15 \mathrm{~nm}$ and an interparticle distance between 30 and $140 \mathrm{~nm}$. These nanopatterns show an enormous mechanical stability and there is no indication for cluster movement or coagulation. ${ }^{197}$ They need no protective coating and are freely accessible to chemical and physical interaction. For the conventional block-copolymer micelle lithography, the only requirements for the substrate material are flatness and resistance to plasma. Thus, a large number of substrates can be used. Using the micellar film as a negative e-beam resist allows for the patterning of flat and electrically conductive substrates such as $\mathrm{Si}, \mathrm{GaAs}$, or $\mathrm{TiO}_{2}{ }^{193}$
An additional coating of the monomicellar film by a carbon layer before the e-beam exposure makes this variation also feasible for non-conductive substrates. ${ }^{197}$

\subsubsection{Bio-Relevant Applications of Block-Copolymer Lithography}

Block-copolymer lithography has a wide range of applications. It has been used, for example, for the fabrication of gold nanowires, ${ }^{198}$ for carbon nanotube growth, ${ }^{199}$ and for patterning nanoparticles. ${ }^{200}$ The micellar film can also be applied as a template for etching. ${ }^{201,202}$ In addition, there are several interesting applications regarding the field of biology. The small size of the nanodots makes them the perfect anchors for individual macromolecules, e.g., for separation, location and screening of DNA and proteins. Functionalized, cell-adhesive nanodots can also be used to study the immobilization of single proteins and their interaction with living cells. Kumar et al. used this technique to produce templates for the selective adsorption of proteins. The preferential interaction of the protein with one of the two polymer segments and the self-organizing of the model protein allows for spatial control in the nanometer scale. This approach may further enable facile, nanometerspaced protein localization. ${ }^{203}$ Another example is the separation of DNA: DNA electrophoresis on nanopatterned surfaces allows for the separation of DNA without separation media, which can degrade the sample and is difficult to handle in small doses. The nanopatterns created by block-copolymer micelle lithography are in the size range of two to three DNA persistence lengths and can be optimized for specific DNA geometries. This approach allows for the separation of a broad band of DNA without a loss in resolution. ${ }^{204}$ Polymer demixing materials can also be used to generate topography on the sample surface, such as islands, pits and ribbons, which can be used to study cell behavior as a function of nanotopography. ${ }^{41}$ Dalby et al. ${ }^{26}$ have shown that nanoislands provoke specific morphology and cytoskeleton responses of endothelial cells cultivated on a polymer demixed topography.

Block copolymer micelle lithography has enabled other novel studies in biology as shown by Spatz et al..$^{30,205}$ In their work, the $\mathrm{Au}$ nanodot pattern provided a means to investigate the universal length scale for integrin clustering and activation, as shown in Figure 14(xiii), which represents the schema of biofunctinalized nanopatterns to control integrin clustering. The $\mathrm{c}(\mathrm{RGDfK})$ functionalized nanodots are separated by a non-fouling background that prevents cell adhesion, such that the cell adhesion is mediated entirely via the functionalized dots. In varying the inter-dot distance, from $28 \mathrm{~nm}$ in (v) and (x) up to $85 \mathrm{~nm}$ in (viii) and (xii), it was first demonstrated that too large a distance between RGD peptides restricted integrin clustering and thereby limited the cell attachment and spreading. 

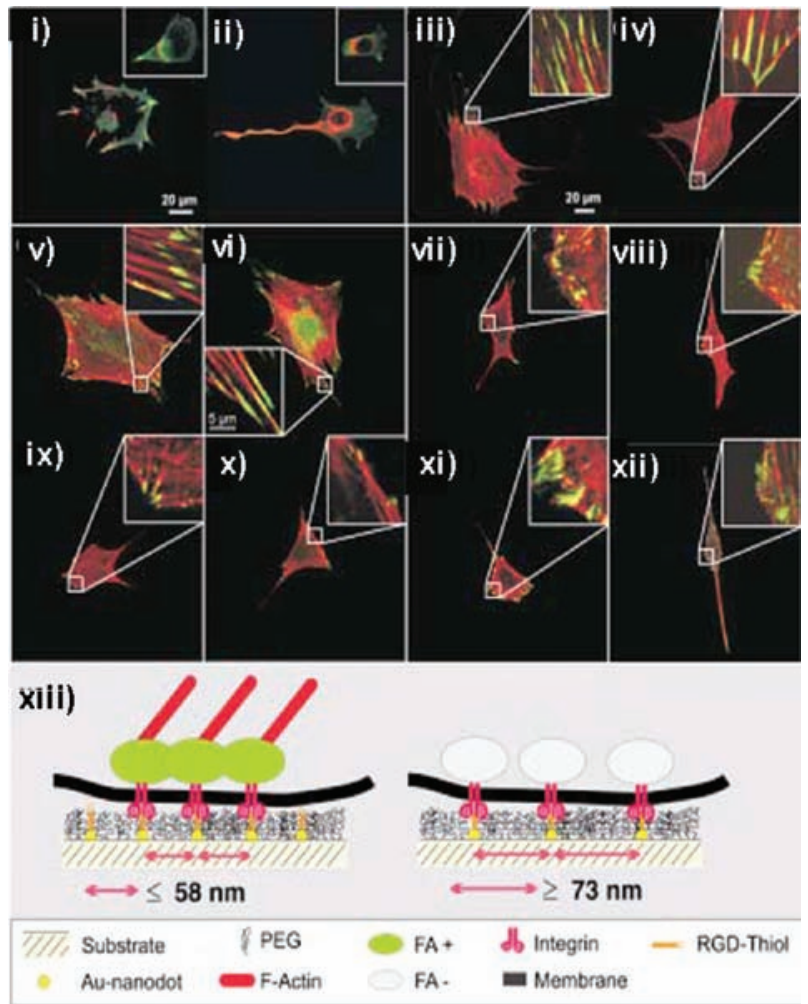

Fig. 14. Fluorescent micrographs of B16-melanocytes expressing GFPintegrin $\beta 3$ (green) and stained for focal adhesion kinase (FAK, red) after $12 \mathrm{~h}$ in culture on block-copolymer nanopatterned substrates with $\mathrm{Au}$ dots at distances of $58 \mathrm{~nm}$ (i) and $73 \mathrm{~nm}$ (ii), with and without (insets) $c($ RGDfK)-thiols. The green spots (i) indicate the clustering of integrin $\beta 3$, the colocalization with FAK shows the existence of multiple focal contacts on the $58 \mathrm{~nm}$ pattern. On the $73 \mathrm{~nm}$ pattern the cell cannot completely spread (ii). Confocal micrographs of MC3T3osteoblasts stained for vinculin (green) and actin (red) after $24 \mathrm{~h}$, homogeneously coated glass cover slip with $\mathrm{c}(\mathrm{RGDfK})$-thiols (iii), fibronectin (iv). Nanopattern with Au-nanodots separated by 28 (v) + (ix), 58 (vi) + (x), 73 (vii) + (xi), and $73 \mathrm{~nm}$ (viii) + (xii). The dots are functionalized with c(RGDfK) (v)-(viii) and without functionalization (ix)-(xii). (xiii) Represents the scheme of biofunctionalized nanopatterns to investigate the influence of integrin clustering in cell membranes. Reprinted with permission from [30], M. Arnold et al., Chemphyschem 5, 383 (2004). (C) 2004, Wiley-VCH.

\subsection{Nanostencil (Shadow Mask Lithography)}

\subsubsection{Nanostencil Lithography Process}

The nanostencil is a tool for resistless lithography. It is based on the deposition of material through miniature shadow masks which consist of silicon nitride. The mask (stencil) is normally produced by conventional processes such as optical or e-beam lithography and ion etching. ${ }^{206-208}$ By supporting the stencil above the desired substrate, a material can then be deposited through the stencil onto the underlying substrate. Figure 15A shows the principle of this patterning method.

A few groups have successfully produced structures $<100 \mathrm{~nm}$ in scale. ${ }^{206,209,210}$ Special set-ups involving collimated evaporation has enabled dots as small as $15 \mathrm{~nm}$

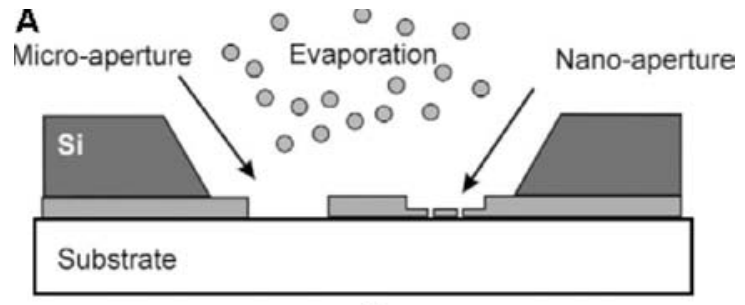

i)

Patterned micro/nano structures

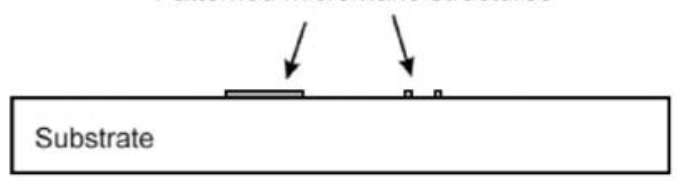

ii)
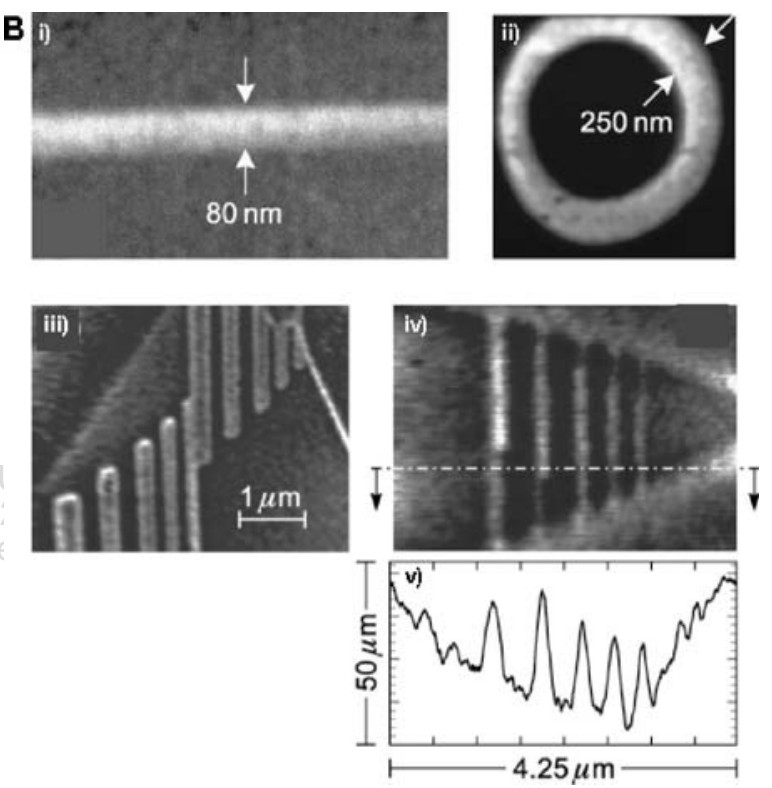

Fig. 15. (A) Schematic diagram of a stencil with different aperture sizes (i); micro- and nano-structures can be deposited in a single-step deposition. After removal of the shadow mask the pattern of the stencil is copied 1:1 onto the substrate (ii). Reprinted with permission from [207], G. M. Kim et al., Microelectronic Engineering 67-8, 609 (2003). () 2003, Elsevier. (B) AFM images of $\mathrm{Cu}$ nanowires produced by nanostencil in the form of a line (i), loop (ii), interdigitated lines (iii), and lines connecting two pads (iv). The height of the line is $10-30 \mathrm{~nm}$. Reprinted with permission from [213], R. Luthi et al., Appl. Phys. Lett. 75, 1314 (1999). (C) 1999, American Institute of Physics.

in diameter or $15-20 \mathrm{~nm}$ in width to be produced. ${ }^{40}$ This method also allows for the combination of nano and micro structures such that, in a single-step deposition, it is possible to produce structures of multiple length scales ranging from sub-100 $\mathrm{nm}$ to several $100 \mu \mathrm{m} \cdot{ }^{207,211}$ This technique in combination with the scanning probe microscope facilitates the controlled movement of the sample relative to the stencil which allows for the fabrication of various patterns with a single mask. ${ }^{212}$ It is also possible to produce complex patterns such as rings and intersecting lines as seen in Figure $15 \mathrm{~B} .^{213}$ 
The target material is not limited to thermal evaporation; material can also be deposited by sputtering, epitaxy, and pulsed laser deposition. ${ }^{214,215}$ Because this technique does not include a photoresist step nor a wet etch step, it can be applied to various substrates including mechanically fragile, chemically active, or organic surfaces. In addition, the contact free deposition of the material reduces the cross-contamination of the sample. The gradual clogging of the apertures that may occur during the deposition process can be reduced by coating the stencil with selfassembled monolayers (SAM). ${ }^{216}$ The small pattern size requires a thin stencil thickness, so the pattern size is also influenced by the stability of the mask. Limitations of this technique are the divergence angle of the deposition beam and diffusion on the surface of the sample.

\subsubsection{Bio-Relevant Applications of the Nanostencil Technique and Summary}

The ability to deposit on virtually any substrate, by contact free nanopattern deposition, makes the nanostencil technique attractive for many bioapplications. Biological studies will certainly profit from the ability to pattern on compliant substrates used, for example, in guided cell growth and tissue engineering. Bucher et al. have already used the nanostencil technique to fabricate arrays of submicron electrodes of both $\mathrm{Au}$ and $\mathrm{TiN}$ for the electrogenic study of cell cultures and tissue slices by extracellular recording and stimulation. ${ }^{217}$ Although microelectrode arrays have enabled electrophysiological studies of cultured excitable cells via measurements of extracellular potentials, nanoelectrode arrays, such as those produced by nanostencilling, will enable much higher resolution and sampling capabilities. ${ }^{31}$

\section{SERIAL NANOPATTERNING TECHNIQUES}

\subsection{E-beam and Focused Ion-Beam Lithography}

For the creation of very small nano-structures $(5-50 \mathrm{~nm})$, direct-writing techniques (such as e-beam lithography (EBL), focused ion-beam lithography (FIBL), and dippen nanolithography (DPN)) proved to be very efficient and successful. The inherent advantages and disadvantages of such techniques are rather obvious, i.e., virtually any nanostructure designed can be fabricated (due to the 'writing' character of these processes), however, these techniques are serial and therefore slow in nature. Thus, EBL (and FIBL) find wide-spread applications in areas where the advantage of creating specific nanostructures outweighs cost and speed considerations. Typical examples are the production of masks and masters for photolithography or microcontact printing, where a single master or mask generally has a long lifetime. However, in the case of e-beam and ion-beam lithography other restrictions might hinder the efficient use in biotechnological systems: cleanroom and vacuum conditions are usually required and often resist and/or etching processes are involved in producing the nanopattern. Furthermore, the technical equipment necessary requires large investments. Dip-pen nanolithography, while approaching the spatial resolution of e-beam and ion-beam lithography, circumvents some of these issues since it is a direct-writing process that works under ambient conditions and the use of aqueous solutions (and biomolecules dissolved therein) as an 'ink' is possible. Furthermore, DPN can potentially be performed by every lab having AFM instrumentation at comparably low costs.

As such, even though progress has been made using EBL or EBL resists to pattern biologically relevant structures, ${ }^{218-220}$ we point the reader to recent reviews on e-beam lithography ${ }^{87,221}$ and ion-beam lithography ${ }^{222}$ and focus in this review on advances in and possibilities with the dip-pen nanolithography techniques for biotechnological applications..$^{23,223}$

\subsection{Dip-Pen Nanolithography (DPN)}

\subsubsection{Mechanism of Dip-Pen Writing}

Dip-pen nanolithography is a direct-writing, scanningprobe-based lithography technique, where an AFM tip is dipped in a solution of molecules of interest and the inked AFM tip then transfers the molecules from the tip to the surface wherever the tip passes, analogous to writing the traditional way with a pen and ink on paper (see Fig. 16) ${ }^{224}$ The molecules are transferred from the tip to the substrate via the liquid meniscus that is forming between the two under ambient conditions. The drivingforce for this process is usually the chemical gradient that develops between the AFM tip and the substrate and, more importantly, the chemical reactivity of the ink molecules with the surface. For this reason early work was conducted on gold substrates with an alkanethiol 'ink', ${ }^{224-226}$ a system well-known for its high chemical affinity and its ability to form well ordered self-assembled monolayers. The feature size in DPN depends on various experimental parameters such as ink composition, substrate roughness, relative humidity, temperature, writing speed, tip geometry, and contact force. While the details of these dependencies are complicated, it is generally found that ink solubility and diffusion processes are key parameters in the formation of DPN patterns. Therefore, increasing temperature (and therefore solubility of the ink molecules) and a higher writing speed (and thus a higher chemical gradient between tip and surface) increases the feature size. ${ }^{227}$ Thus far, feature sizes down to $15 \mathrm{~nm}$ have been demonstrated (see Fig. 16). ${ }^{225}$ This is comparable to traditional serial high-resolution techniques (e-beam lithography, ion-beam lithography, other scanning probe lithography approaches), however, DPN offers the advantage of a larger variety of achievable structures and chemistries that can be patterned 


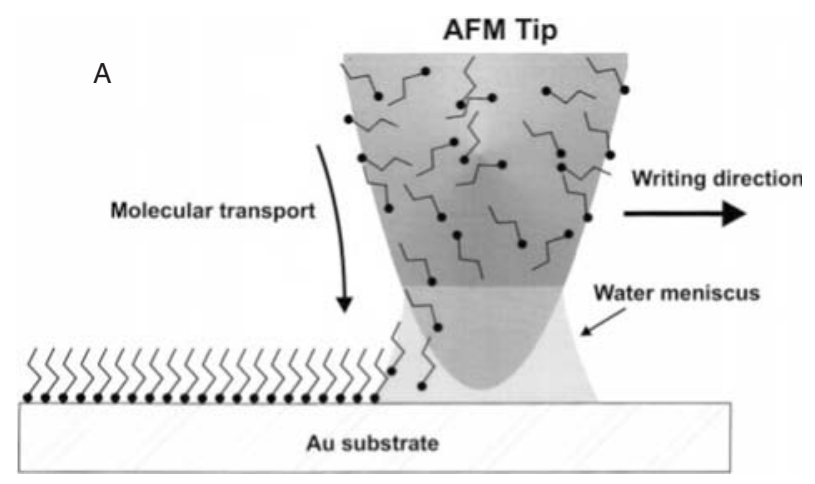

B

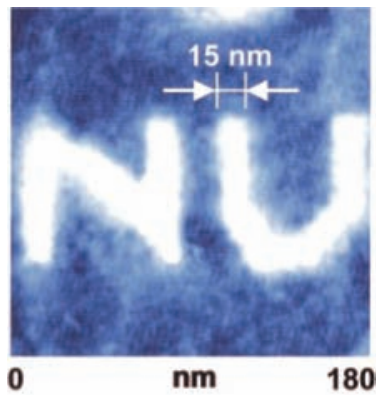

Fig. 16. (A) Schematic representation of DPN. An AFM tip is 'inked' with an alkanethiol solution and brought in contact with a Au surface. A water meniscus forms between the AFM tip and the substrate. Molecules are transported through the meniscus to the surface, thus writing an alkanethiol SAM wherever the tip passes. Reprinted with permission from [224], R. D. Piner et al., Science 283, 661 (1999). (C) 1999 American Association for the Advancement of Science. (B) AFM image of a 16-mercaptohexadecanoic acid SAM written on a Au substrate showing $15 \mathrm{~nm}$ lateral resolution. Reprinted with permission from [225], S. H. Hong et al., Science 286, 523 (1999). (C) 1999, American Association for the Advancement of Science.

and all this under ambient conditions and with the possibility to parallelize the writing process. ${ }^{228}$

Recently, it was also indicated that, contrary to earlier suggestions, ${ }^{224,225,227}$ a water meniscus is not necessary to transfer ink molecules onto the substrate. ${ }^{229,230}$ This opened up the path to pattern even more chemical systems and materials. In fact, a water meniscus (and water present on the surface if relative humidity is not negligible) inhibits the formation of a DPN pattern in certain cases (e.g., if hydrophobic molecules are used). Today, it is thought that the water meniscus does not play a significant role and deposition occurs via surface diffusion of molecules (and is therefore exponentially dependant on temperature) rather then transport through a water meniscus. ${ }^{229,230} \mathrm{Sev}-$ eral other papers are investigating DPN from a theoretical background, analyzing and modeling diffusion processes that lead to the nanopattern formation. ${ }^{231-233}$

The DPN technique also offers some distinct advantages in terms of registration of the nanopatterns and parallelization possibilities. ${ }^{228}$ Since in the DPN process, the same tool is used for writing and imaging it is possible to use multiple inks to achieve nanostructures with chemically pristine patterns of multiple different materials with near perfect alignment and 5-nm spatial separation (see Fig. 17). ${ }^{225}$ In addition, parallelization of the DPN process is feasible by using multiple tips and a suitable feedback control, e.g., a tiltable substrate holder. ${ }^{226}$ Salaita et al. have further extended the idea of parallel writing of DPN nanostructures with commercial 26-pen arrays and custom made 250-pen arrays (see Fig. 17). ${ }^{234}$ Very recently, Wang and $\mathrm{Liu}^{235}$ incorporated the dip-pen nanolithography technique together with other cantilever based imaging and patterning methods (scanning probe contact printing, atomic, and lateral force microscopy) on a multifunctional probe array, thereby enabling a rich variety of operations with minimal chemical crosstalk and high registration accuracy to be executed. Their work illustrates that DPN has great potential for the large scale, multicomponent fabrication of nanoscale features.

Since its invention in 1999, DPN has been applied to a multitude of systems other than alkanethiols on gold: pattering different dyes, ${ }^{236}$ dendrimers, ${ }^{237}$ photosensitive conducting polymers, ${ }^{238}$ aqueous metals nanocrystal dispersions, ${ }^{239,240}$ or metallic nanostructures using aqueous metal salt inks whose metal ions are reduced on the surface electrochemically to form a metallic nanopattern extending DPN to electrochemical AFM nanolithography ${ }^{241-243}$ or using a DPN alkanethiol SAM as a mask for etching $3 \mathrm{D}$ structures into a gold covered silicon substrate, ${ }^{244}$ just to name a few. A comprehensive review of investigated DPN systems was published recently by Ginger, Zhang, and Mirkin. ${ }^{23}$ ishers

\subsubsection{Bioapplications of DPN}

In this review, we focus on advances in DPN that are or might be relevant for nanobiotechnological applications. DPN is of high interest in this field because of some inherent advantages discussed above: it is a maskless lithography method, parallelization of DPN can be achieved, and it is performed under ambient conditions. These features make it attractive for the pattering of biological species (proteins, DNA, nucleic acid, cells). Furthermore, DPN was also shown to work in tapping mode, rather than contact mode. ${ }^{245,246}$ Using tapping mode for DPN exerts less force on the patterning system and could therefore be used as an even 'gentler' way to deposit biomolecule nanostructures since surface scratching and dragging of biomolecules are reduced. ${ }^{247}$

Indeed, a variety of publications have become available recently that report the fabrication of biologically relevant nanostructures with different approaches regarding the kind of biomolecules and the immobilization strategies used to nanopattern the biological function. ${ }^{167,245,246,248-255}$

Demers et al. used DPN to directly pattern hexanethiolmodified oligonucleotides to a gold substrate with a feature size down to $50 \mathrm{~nm}$. The hexanethiol then chemisorbs to the gold substrate, anchoring the oligonucleotides to the surface. Backfilling of the bare gold substrate with a 


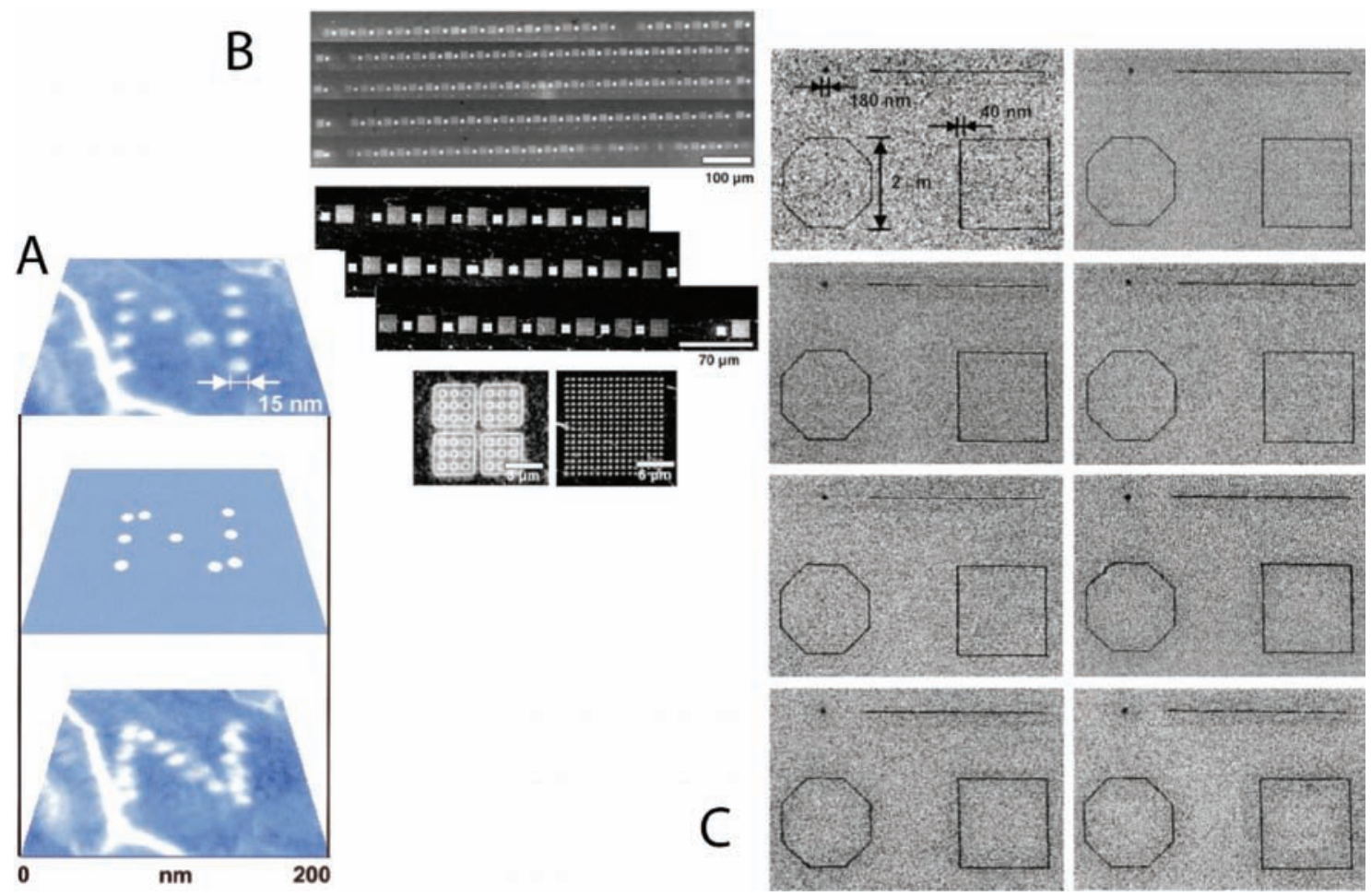

Fig. 17. (A) Lateral force microscopy images of 16-mercaptohexadecanoic acid SAM dots on Au. Middle image shows the anticipated position of dots printed in a second run calculated from the first set of points. The last image in the series shows the second set of dots printed within the first set of dots showing the high registration possibilities offered by DPN. Reprinted with permission from [225], S. H. Hong et al., Science 286, 523 (1999). (C) 1999, American Association for the Advancement of Science. (B) Optical and SEM images of a 130 grid and dot array produced simultaneously by using 130 AFM tips in parallel. Reprinted with permission from [234], K. Salaita et al., Small 1, 940 (2005). (C) 2005, American Association for the Advancement of Science. (C) Lateral force microscopy images also produced simultaneously using eight writing and one imaging tip with an alkanethiol ink on Au. Reprinted with permission from [226], S. Hong and C. A. Mirkin, Science 288, 1808 (2000). () 2000, American Association for the Advancement of Science.

hydrophobic 1-octadecanethiol SAM passivates the background towards unspecific DNA adsorption. Subsequently, $13 \mathrm{~nm} \mathrm{Au}$ colloids coated with the complementary DNA strand were attached to the DPN pattern, showing high specificity and little unspecific adsorption. An alternative approach using different chemistry even allows for DNA patterning of oxidized silicon wafers, thus avoiding problems arising from the conductive character of the gold substrate (charge-transfer or near-field optical phenomena studies are not possible) (see Fig. 18). ${ }^{249}$ Alternatively, DNA molecules were also grafted to a DPN surface pattern via an indirect coupling process. In that case, alkylamine-terminated DNA residues were bound to 16mercaptohexadecanoic acid pattern by the formation of an amide bond. Again, these surface-bound DNA strands were used to bind Au colloids containing the complementary DNA strand to the substrate surface. ${ }^{248}$ Nyamjav and Ivanisevic patterned charged polyelectrolytes using DPN and showed that these structures can be used to adsorb the DNA strands in a stretched and aligned matter in order to develop a methodology to localize bound DNA molecules in complex architectures. ${ }^{250}$

Patterning of proteins at the nanoscale scale without loosing the activity is an inherently more difficult task.
Wilson et al. used thiolated collagen and collagen-like peptides to directly pattern lines as small as $30-50 \mathrm{~nm}$ in width as well as much larger structures. ${ }^{245}$

Lysozyme and IgG proteins were patterned with dot sizes down to $45 \mathrm{~nm}$ by a direct-write process. ${ }^{253}$ In this case, native protein versions could be used, but the AFM tip was modified with a thiotic acid coating to facilitate inking the tip with proteins. Direct-writing of the proteins was then performed at relatively high humidity (low humidity lead to inconsistent transport properties) and the substrate background was passivated with PEG. On the same sample, both lysozyme and IgG patterns could be generated showing the possibility to fabricate complex multicomponent nanostructures without crosscontamination. Exposing such a sample to an anti- $\operatorname{IgG}$ solution led to an increase in the height of the $\operatorname{IgG}$ spots but not of the lysozyme dots, showing that (a) the IgG protein is still active and (b) that no cross-contamination or unspecific adsorption could be observed. ${ }^{253}$

In an indirect way, Lee et al. produced very similar protein nanoarrays of lysozyme and IgG with very little unspecific background adsorption and feature sizes down to $100 \mathrm{~nm}$. Specifically, 16-mercaptohexadecanoic acid was used in the dip-pen nanolithography process to create 
A i)
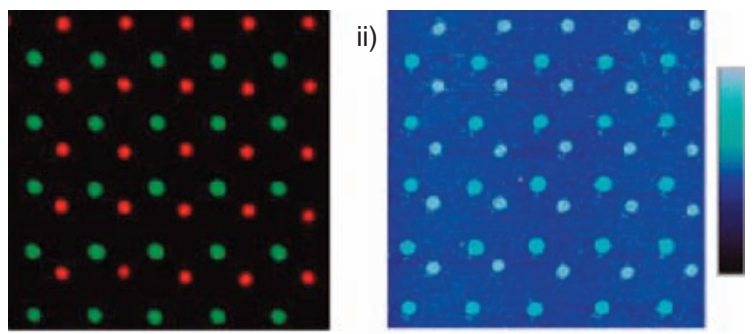

B

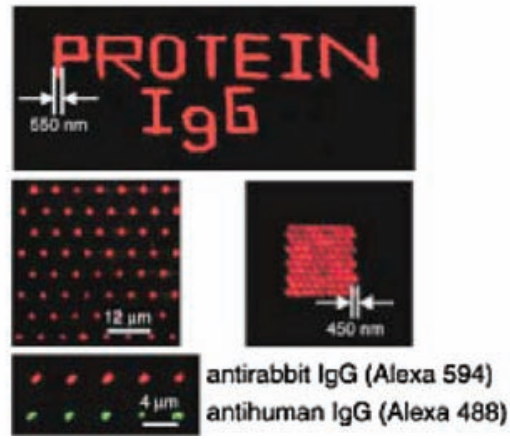

Fig. 18. Examples of direct-patterning of DNA (A) and proteins (B) using DPN: (A) Direct patterning of multiple DNA inks. Combined red-green epifluorescence image (i) of two different fluorophore-labeled DNA sequences simultaneously hybridized to a two-DNA-sequence array deposited on a $\mathrm{SiO}_{x}$ substrate by DPN. Tapping-mode AFM image (ii) of 5 (dark)- and 13 (light)-nm gold nanoparticles assembled on the same pattern (as seen in (i) after dehybridization of the fluorophorelabeled DNAB) Fluorescence images of DPN-generated antirabbit $\mathrm{IgG}$ (labeled withAlexa 594) structures on a $\mathrm{SiO}_{2} /$ surface. Reprinted with permission from [249], L. M. Demers et al., Science 296, 1836 (2002). C 2002, American Association for the Advancement of Science. (B) Patterning of proteins. Bottom left of (B): two-component protein array on an aldehyde-derivatized $\mathrm{SiO}_{2}$ surface. Reprinted with permission from [256], J. H. Lim et al., Angew. Chem.-Int. Ed. 42, 2309 (2003). (C) 2003, Wiley-VCH.

the patterns and 11-mercaptoundecyl-tri(ethylene glycol) was used to backfill the exposed substrate regions. Proteins show a relatively high affinity for the carboxylic acidterminated monolayers at $\mathrm{pH} \mathrm{7,} \mathrm{while} \mathrm{the} \mathrm{ethylene} \mathrm{glycol}$ background is resistant to protein adsorption. By exposing such a patterned surface to either lysozyme or IgG, the proteins specifically adsorbed to the regions containing the carboxylic acid and no adsorption was observed on the ethylene glycol background. ${ }^{251}$ They again observed an increase in the pattern height after exposing an $\mathrm{IgG}$ nanoarray to a solution containing anti-IgG proteins, indicating binding of the anti-IgG to the IgG on the array, thus illustrating that the proteins retained their activity after adsorption. Furthermore, after patterning a cellular adhesion protein (retronectin) on $200 \mathrm{~nm}$ spots separated by $700 \mathrm{~nm}$, it was observed that 3T3 Swiss fibroblast cells preferentially adsorbed to patterned areas. This shows that submicrometer features can support cell adhesion. ${ }^{251}$ More generally, it shows the importance of having suitable tools to systematically identify the relationship between nanofeature size and composition in studies of cell adhesion and other related processes.
Another system of practical importance in biomolecular recognition processes is the streptavidin-biotin system. Hyun et al. showed a DPN patterning approach that produces biotinylated structures in an inert background. ${ }^{252}$ To do so, a COOH-terminated SAM is produced by DPN and the background is rendered non-interactive for proteins by backfilling it with an ethylene glycol terminated thiol SAM. Then, the COOH-groups in the patterned SAM were converted to reactive $N$-hydroxysuccinimide ester, followed by reaction with $\mathrm{NH}_{2}$-biotin. This covalently links the biotin molecules to the patterned SAM. After activating the biotinylated areas with streptavidin, this system poses the possibility to conjugate any biotin-containing protein or species to this kind of nano-pattern. Hyun et al. showed the feasibility of this approach by binding biotinylated BSA to this kind of DPN pattern. ${ }^{252}$ This system has the advantage of promising much better control over the interactions between the (biotinylated) proteins and the substrate, since it relies on a specific binding chemistry. Other approaches that directly pattern proteins or adsorb proteins on DPN nanostructures are potentially more prone to denaturing problems that might be of special importance when it comes to few/single protein arrays, wherein exact positioning and an intact protein structure will be of great importance.

Another well-known molecular recognition system was used by Agarwal et al. to pattern histidine-tagged proteins on nickel surfaces. ${ }^{246}$ They used the strong affinity between nickel ions and histidine in a DPN process in the presence of an electric field, as introduced earlier. ${ }^{241,242}$ Applying a potential between the probe and the nickel coated substrate through the water meniscus results in the ionization of the nickel surface and histidine-tagged proteins are then bound to the nickel ions on the surface.

A very general and thus potentially powerful approach to direct-write proteins on modified silicon oxide surfaces was proposed by Lim et al. (see Fig. 18). ${ }^{256}$ In their case, the AFM tip is modified with a Si-PEG layer. This layer inhibits protein adsorption to the tip and due to the hydrophilic character of this coating also inhibits denaturing of the proteins. Since the proteins are not interacting with the tip, activation energies for the transport from the tip to the surface are also very favorable. In addition, this coating is also responsible for much better wetting of the tip with the ink compared to standard $\mathrm{Si}_{3} \mathrm{~N}_{4}$ tips. In a next step, the silicon dioxide surface is modified to better interact with the proteins coming from the ink. This is done either by treating the surface with base (creating negative charges that interact with positive patches of the proteins) or by creating an aldehyde-modified surface which interacts via amine groups of the proteins. ${ }^{256}$ In this case, crosscontamination between human and rabbit IgG was low and selectivity and activity of the patterned $\mathrm{IgG}$ proteins remained intact, proving that direct-writing of proteins on (modified) silicon dioxide surfaces down to about $55 \mathrm{~nm}$ is feasible today. 
Noy and co-workers pushed the limits of DPN by combining it with scanning optical confocal microscopy to fabricate and visualize luminescent nanoscale patterns. By doing so, they were able to control the deposition of single molecules and they could produce and visualize protein patterns on surfaces. ${ }^{257}$

A variation of DPN and its immediate predecessor is termed nanografting or nanoshaving, introduced by $\mathrm{Xu}$ and Liu in 1997 (a schematic overview is given in Fig. 19). ${ }^{258}$ In this technique, a monolayer of a molecule (usually
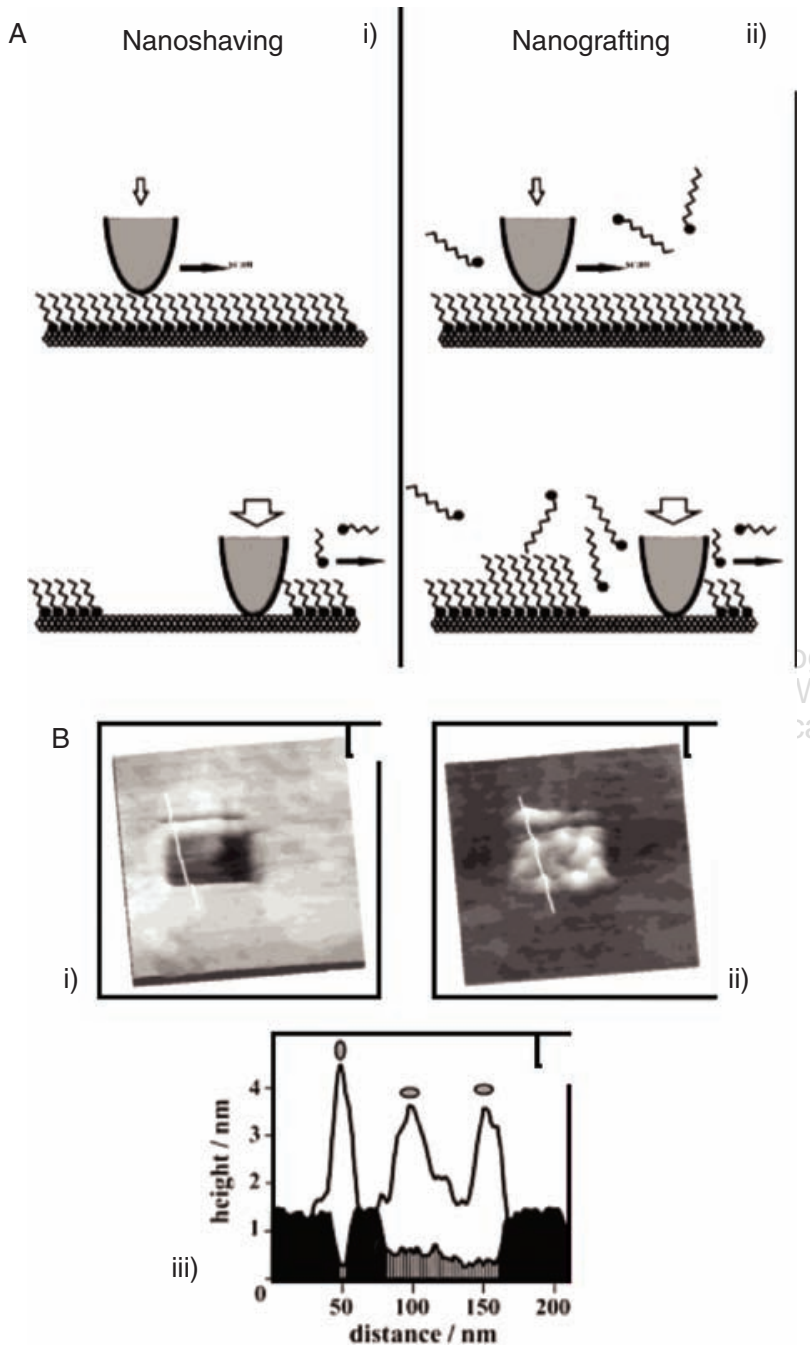

Fig. 19. (A) Schematic representation of the nanoshaving (i) and the nanografting (ii) approaches. In the first, an AFM tip is run across the surface at high loads, shaving off the initial SAM. In the latter, the process is similar, but the 'shaved' regions are backfilled with a different 'ink' molecule. Reprinted with permission from [260], G. Y. Liu et al., Acc. Chem. Res. 33, 457 (2000). (C) 2000, American Chemical Society. (B) Patterning of lysozyme using the nanografting technique: two regions were nanografted by replacing the initial SAM with a (shorter) carboxylterminated SAM (i). Then, lysozyme was selectively adsorbing only to the carboxylated areas. The pattern thus extends out of the SAM in the background (ii). Line scan of (iii) revealing the conformational structure of the lysozyme molecules adsorbed to the surface. Reprinted with permission from [259], K. Wadu-Mesthrige et al., Langmuir 15, 8580 (1999). (C) 1999, American Chemical Society. a thiol SAM on gold $^{258-263}$ ) is adsorbed on the substrate and an AFM tip is used (at high loads) to locally 'shave' the monolayer (using loads higher then the displacement threshold of the SAM molecules), hence the term nanoshaving. ${ }^{260}$ When this shaving is performed in solution, free molecules of a different species can then adsorb to the cleaved areas and thereby create chemical nanopatterns, this process is then commonly referred to as nanografting. ${ }^{258,260}$ A very similar concept was presented by Amro et al. who termed their process nanopen reader and writer (NPRW). ${ }^{264,265}$ This process is a combination of nanoshaving and DPN, where an AFM tip coated with an ink first removes a SAM on the surface using high loads (nanoshaving) and then directly writes onto the now uncovered areas (DPN). Structures of only 32 alkanethiol molecules could be achieved by this process resulting in a single dot of $2 \times 4 \mathrm{~nm}^{2}$ (Ref. [265]).

A very similar concept was introduced by Harnett et al. who used e-beam lithography to locally remove a patterned SAM and backfilled these patches with different, reactive SAMs to introduce (biological) functions in these spots. ${ }^{266}$

Wadu-Mesthrige used nanografting already in 1999 to adsorb lysozyme onto a chemical nanopattern. ${ }^{259}$ They first formed an hydrophobic alkanethiol SAM, then a COOH-terminated SAM was nanografted into the initial SAM. Lysozyme was then adsorbed onto the carboxylic endgroups of the nanopattern via electrostatic interactions. They also used aldehyde-terminated SAMs for the nanografting procedure to which lysozyme or IgG proteins could then be adsorbed via imine bonds. Although they observed some protein adsorption onto the hydrophobic background SAM, these proteins could be removed by washing with a $1 \%$ tween- 20 solution. Liu et al. ${ }^{263}$ used the same nanografting approach to create DNA nanopattern on gold in an alkanethiol SAM by shaving the SAM and replacing the shaved areas with thiolated single-stranded DNA molecules. The structure of the nanopattern could be observed in situ with AFM and the activity of the ssDNA molecules was checked by an enzyme digestion experiment. A good early review on nanografting and nanoshaving (of SAMs) was written by Liu et al. ${ }^{260}$

\subsubsection{Summary of $D P N$}

In conclusion, dip-pen nanolithography is a very versatile direct-writing nanopatterning technique with high potential for answering nanobiotechnological questions. Patterning of proteins and DNA with good spatial resolution is possible today (around $100 \mathrm{~nm}$ ). It can, however, not only be used to for the patterning of biomolecules but also for the generation of chemical templates for the study of biorecognition processes. This is also due to one major advantage of DPN, that is that commonly available surface engineering techniques (thiol-based chemistry on Au surfaces, pegylation of surfaces to prevent unspecific protein adsorption, DNA anchoring techniques, etc.) can be used to 


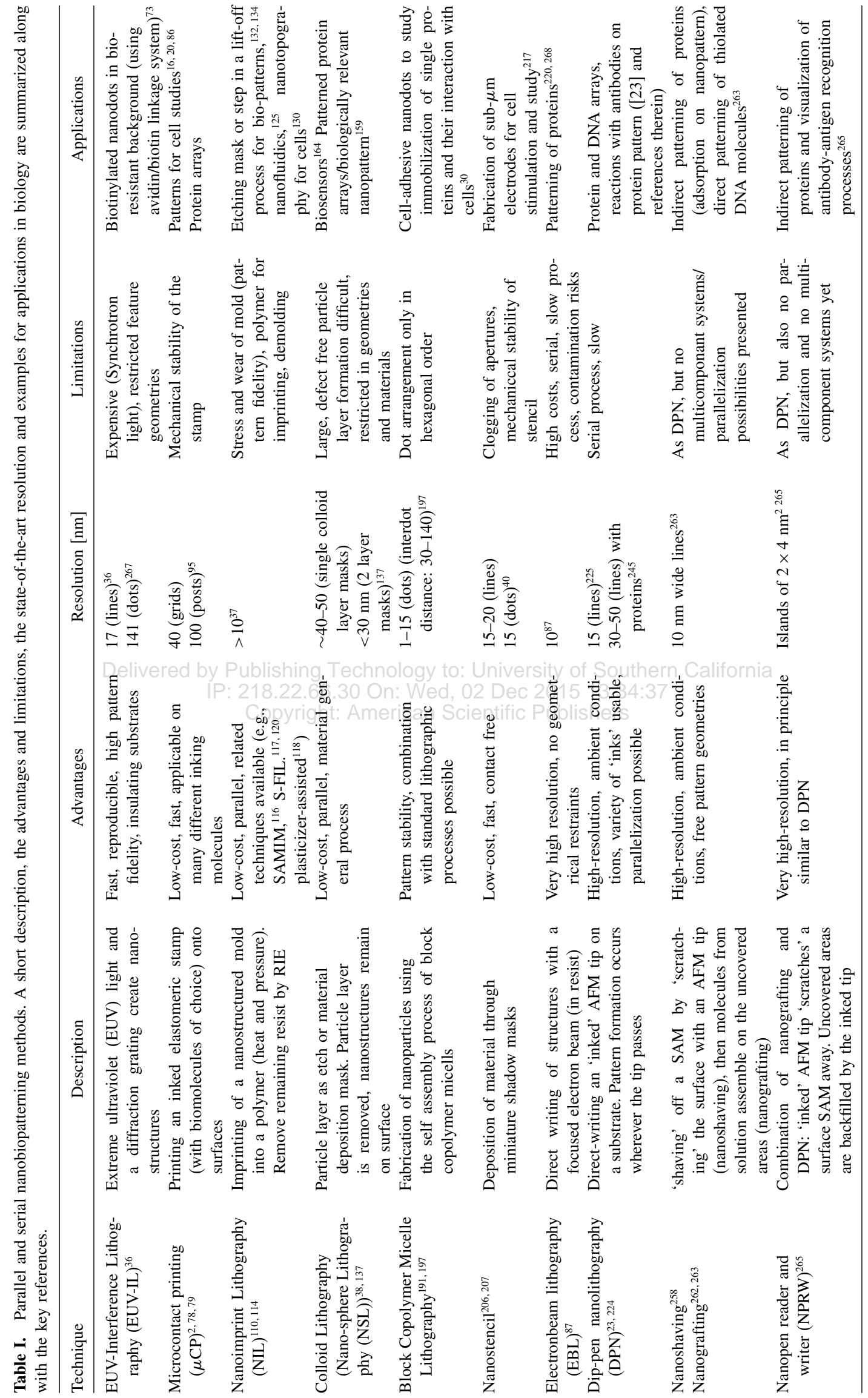


structure biologically relevant patterns in the nanometer range. Still, progress has to be made in terms of spatial resolution of biological structures to reach values presented by standard DPN with alkanethiols on gold $(\sim 15 \mathrm{~nm})$. Being able to structure biological molecules in these dimensions would mean to reach the size of individual biological macromolecules and offer the potential to investigate individual molecular recognition processes of interest to a range of applications from single molecules to cell-surface interactions. Indeed, first data is published on experiments with cells on samples structured by DPN studying the reactions of cells with true nanochemical pattern. One possible way to reach these resolutions might be in the combination with some of the presented techniques like nanografting and DPN or the use of sharper tips (e.g., carbon nanotubes).

On the other hand, while a serial process in nature, advances in parallelization and registration of patterns of biomolecules have been presented that will also permit the use of DPN in biosensor array technology. In this case, DPN might offer unique opportunities in the production and registration of multicomponent nanoarrays of biomolecules.

\section{CONCLUSIONS AND OUTLOOK}

The discovery of light-microscopy during the 17 th century has changed our attitude towards the 'micro-world', but it was not until the 20th century, when the appearance of microelectronics provided the tools to overcome the observer status, that we were able to start manipulating objects (some of them living) at the micron scale. Similarly, the Nobel-prize winning inventions of electron microscopy in 1931 and scanning tunneling microscopy in 1981 allowed us to look into the 'nano-world'. The images provided by these new technologies, particularly in biology, made us quickly realize that we needed new tools to access the individual building blocks of living organisms (e.g., proteins, DNA, and supramolecular complexes) in order to hope for a better understanding of the complicated processes of life.

The techniques described in this review (and summarized in Table I) provide us with new ways to ask novel questions and address specific properties at the lengthscale of single biomolecules. Their impact is expected to be greatest, first, in the field of biosensing because these nanopatterning techniques promise to further increase the information density of array-based biosensors; provide the possibility for the development of a new generation of biosensors that make use of novel nano-physical principles, e.g., the localized surface plasmon resonance; and allow for the production of well-defined single biomolecule arrays. In addition, nanobiopatterns are expected to revolutionize our understanding of cell-surface interactions and as such might contribute to the development of better biomaterials and to tissue engineering.
At present, the parallel nanobiopatterning methods (Section 2) can provide large scale homogenous samples with usually repetitive patterns. The serial writing methods (Section 3) are more flexible with the type of pattern but generally slow when producing large areas. Future challenges will include the production of inhomogeneous patterns without which no meaningful biosensing nanoarray can be imagined and only the simplest biological questions can be asked. All of the current nanobiopatterning techniques have their roots in semiconductor industrial processes and as such they are two dimensional in nature. On the other hand, true three dimensional control would be required in order to fully mimic the complex environment of cells and to ask questions such as 'what is the effect of certain signals on the differentiation, proliferation, or apoptosis of cells'. Although, such control is not yet foreseen, the existing nanobiopatterning techniques described in this review already provide us with many possibilities to learn more about biology in a novel fashion.

Acknowledgments: The authors would like to acknowledge Prof. Marcus Textor for both scientific and financial support and Ms. S. Frerker for EUV-IL AFM images. EUV Interference lithography exposures reported in this work were performed at the Swiss Light Source, Paul Scherrer Institut, Villigen, Switzerland. For funding, the authors acknowledge the ETH Zurich, EPF Lausanne and the National Science and Engineering Council of Canada (NSERC). For specific project funding, the authors acknowledge the European Science Foundation EUROCORES Programme for the 'Self-Organised Nanostructures' (SONS; NanoSMAP) project support from the Swiss National Science Foundation (SNF) and the European Commission (EC) Sixth Framework Programme, EC funding is also acknowledged for the NANOCUES project (FP6-NMP-2002-3.4.1.2.-1.), and the Swiss funding program on nanotechnology is acknowledged for funding in the Top Nano 21 (Project 5971.2) as well as the KTI Swiss Innovation Promotion Agency.

\section{References and Notes}

1. Y. N. Xia and G. M. Whitesides, Angew. Chem.-Inte. Ed. 37, 551 (1998).

2. G. M. Whitesides, E. Ostuni, S. Takayama, X. Y. Jiang, and D. E. Ingber, Ann. Rev. Biomed. Engin. 3, 335 (2001).

3. A. C. R. Grayson, R. S. Shawgo, A. M. Johnson, N. T. Flynn, Y. W. Li, M. J. Cima, and R. Langer, Proceedings of the IEEE 92, 6 (2004).

4. S. P. A. Fodor, J. L. Read, M. C. Pirrung, L. Stryer, A. T. Lu, and D. Solas, Science 251, 767 (1991).

5. G. Ramsay, Nature Biotechnol. 16, 40 (1998)

6. D. Figeys and D. Pinto, Electrophoresis 22, 208 (2001).

7. K. N. Lee, D. S. Shin, Y. S. Lee, and Y. K. Kim, J. Micromechan. Microengin. 13, 474 (2003)

8. I. Shin, S. Park, and M. R. Lee, Chemistry-a European Journal 11, 2894 (2005).

9. V. I. Chin, P. Taupin, S. Sanga, J. Scheel, F. H. Gage, and S. N. Bhatia, Biotechnol. Bioengineer. 88, 399 (2004). 
10. C. S. Chen, X. Y. Jiang, and G. M. Whitesides, Mrs Bulletin 30 , 194 (2005).

11. A. Revzin, R. G. Tompkins, and M. Toner, Langmuir 19, 9855 (2003).

12. K. S. Lam and M. Renil, Current Opinion in Chemical Biology 6, 353 (2002)

13. M. Krishnan, V. Namasivayam, R. S. Lin, R. Pal, and M. A. Burns, Current Opinion in Biotechnology 12, 92 (2001).

14. E. Verpoorte and N. F. De Rooij, Proceedings of the IEEE 91, 930 (2003).

15. L. Y. Koo, D. J. Irvine, A. M. Mayes, D. A. Lauffenburger, and L. G. Griffith, J. Cell Sci. 115, 1423 (2002).

16. C. S. Chen, M. Mrksich, S. Huang, G. M. Whitesides, and D. E. Ingber, Science 276, 1425 (1997).

17. D. S. Gray, J. Tien, and C. S. Chen, J. Biomed. Mater. Res. Part A 66A, 605 (2003).

18. S. Raghavan and C. S. Chen, Adv. Mater. 16, 1303 (2004).

19. M. Scholl, C. Sprossler, M. Denyer, M. Krause, K. Nakajima, A. Maelicke, W. Knoll, and A. Offenhausser, J. Neurosci. Methods 104, 65 (2000).

20. A. A. Oliva, C. D. James, C. E. Kingman, H. G. Craighead, and G. A. Banker, Neurochem. Res. 28, 1639 (2003).

21. G. Vozzi, C. Flaim, A. Ahluwalia, and S. Bhatia, Biomaterials 24 , 2533 (2003)

22. D. B. Weibel, P. Garstecki, and G. M. Whitesides, Current Opinion in Neurobiology 15, 560 (2005).

23. D. S. Ginger, H. Zhang, and C. A. Mirkin, Angew. Chem. Int. Ed. 43, 30 (2004)

24. N. L. Rosi and C. A. Mirkin, Chem. Rev. 105, 1547 (2005).

25. A. Curtis and C. Wilkinson, Trends in Biotechnology 19, 97 (2001).

26. M. J. Dalby, M. O. Riehle, H. Johnstone, S. Affrossman, and A. S. G. Curtis, Biomaterials 23, 2945 (2002).

27. A. Thapa, T. J. Webster, and K. M. Haberstroh, J. Biomed. Mater. Res. Part A 67A, 1374 (2003).

28. N. W. Karuri, S. Liliensiek, A. I. Teixeira, G. Abrams, S. Campbell, P. F. Nealey, and C. J. Murphy, J Cell Sci. 117, 3153 (2004).

29. G. Maheshwari, G. Brown, D. A. Lauffenburger, A. Wells, and L. G. Griffith, J. Cell Sci. 113, 1677 (2000).

30. M. Arnold, E. A. Cavalcanti-Adam, R. Glass, J. Blummel, W. Eck, M. Kantlehner, H. Kessler, and J. P. Spatz, Chem. Phys. Chem. 5, 383 (2004)

31. L. Berdondini, P. D. van der Wal, O. Guenat, N. F. de Rooij, M. Koudelka-Hep, P. Seitz, R. Kaufmann, P. Metzler, N. Blanc, and S. Rohr, Biosensors \& Bioelectronics 21, 167 (2005).

32. Y. Chen, G. Y. Jung, D. A. A. Ohlberg, X. M. Li, D. R. Stewart, J. O. Jeppesen, K. A. Nielsen, J. F. Stoddart, and R. S. Williams, Nanotechnology 14, 462 (2003).

33. G. F. Zheng, F. Patolsky, Y. Cui, W. U. Wang, and C. M. Lieber, Nature Biotechnology 23, 1294 (2005).

34. B. D. Gates, Q. B. Xu, M. Stewart, D. Ryan, C. G. Willson, and G. M. Whitesides, Chem. Rev. 105, 1171 (2005).

35. M. Geissler and Y. N. Xia, Adv. Mater. 16, 1249 (2004).

36. H. H. Solak, D. He, W. Li, S. Singh-Gasson, F. Cerrina, B. H. Sohn, X. M. Yang, and P. Nealey, Appl. Phys. Lett. 75, 2328 (1999).

37. S. Y. Chou, P. R. Krauss, and P. J. Renstrom, Science 272, 85 (1996).

38. P. Hanarp, D. Sutherland, J. Gold, and B. Kasemo, Nanostructured Mater. 12, 429 (1999).

39. M. Park, C. Harrison, P. M. Chaikin, R. A. Register, and D. H. Adamson, Science 276, 1401 (1997).

40. M. M. Deshmukh, D. C. Ralph, M. Thomas, and J. Silcox, Appl. Phys. Lett. 75, 1631 (1999).

41. R. Barbucci, D. Pasqui, A. Wirsen, S. Affrossman, A. Curtis, and C. Tetta, J. Mater. Sci.-Mater. Medicine 14, 721 (2003).

42. R. G. Flemming, C. J. Murphy, G. A. Abrams, S. L. Goodman, and P. F. Nealey, Biomaterials 20, 573 (1999).
43. J. W. Carlson, T. Bayburt, and S. G. Sligar, Langmuir 16, 3927 (2000).

44. P. Lenz, C. M. Ajo-Franklin, and S. G. Boxer, Langmuir 20, 11092 (2004).

45. K. Morigaki, K. Kiyosue, and T. Taguchi, Langmuir 20, 7729 (2004).

46. F. F. Rossetti, M. Bally, R. Michel, M. Textor, and I. Reviakine, Langmuir 21, 6443 (2005).

47. A. Dahlin, M. Zach, T. Rindzevicius, M. Kall, D. S. Sutherland, and F. Hook, J. Am. Chem. Soc. 127, 5043 (2005).

48. P. Kingshott and H. J. Griesser, Current Opinion in Solid State \& Materials Science 4, 403 (1999).

49. J. L. Dalsin, L. J. Lin, S. Tosatti, J. Voros, M. Textor, and P. B. Messersmith, Langmuir 21, 640 (2005).

50. J. L. Dalsin, B. H. Hu, B. P. Lee, and P. B. Messersmith, J. Am. Chem. Soc. 125, 4253 (2003).

51. M. Amiji and K. Park, Biomaterials 13, 682 (1992).

52. E. Ostuni, R. G. Chapman, R. E. Holmlin, S. Takayama, and G. M. Whitesides, Langmuir 17, 5605 (2001).

53. J. P. Bearinger, D. G. Castner, S. L. Golledge, A. Rezania, S. Hubchak, and K. E. Healy, Langmuir 13, 5175 (1997).

54. G. L. Kenausis, J. Voros, D. L. Elbert, N. P. Huang, R. Hofer, L. Ruiz-Taylor, M. Textor, J. A. Hubbell, and N. D. Spencer, J. Phys. Chem. B 104, 3298 (2000).

55. J. D. Le, Y. Pinto, N. C. Seeman, K. Musier-Forsyth, T. A. Taton, and R. A. Kiehl, Nano Lett. 4, 2343 (2004).

56. B. Samori and G. Zuccheri, Angew. Chem.-Int. Ed. 44, 1166 (2005).

57. J. Voros, T. Blattler, and M. Textor, Mrs Bulletin 30, 202 (2005).

58. B. D. Gates, Q. B. Xu, J. C. Love, D. B. Wolfe, and G. M. Whitesides, Ann. Rev. Mater. Res. 34, 339 (2004).

59. T. Ito and S. Okazaki, Nature 406, 1027 (2000).

60. D. Falconnet, A. Koenig, F. Assi, and M. Textor, Adv. Funct. Mater. 14, 749 (2004)

61. R. Michel, J. W. Lussi, G. Csucs, I. Reviakine, G. Danuser, B. Ketterer, J. A. Hubbell, M. Textor, and N. D. Spencer, Langmuir 18, 3281 (2002).

62. M. A. Holden, S. Y. Jung, and P. S. Cremer, Anal. Chem. 76, 1838 (2004).

63. L. A. Chrisey, C. E. OFerrall, B. J. Spargo, C. S. Dulcey, and J. M. Calvert, Nucleic Acids Research 24, 3040 (1996).

64. G. V. Shivashankar and A. Libchaber, Appl. Phys. Lett. 73, 417 (1998).

65. H. B. Yin, T. Brown, J. S. Wilkinson, R. W. Eason, and T. Melvin, Nucleic Acids Research 32 (2004).

66. H. H. Solak, C. David, J. Gobrecht, V. Golovkina, F. Cerrina, S. O. Kim, and P. F. Nealey, Microelectronic Engineering 67-8, 56 (2003).

67. H. H. Solak, C. David, J. Gobrecht, L. Wang, and F. Cerrina, Microelectronic Engineering 61-2, 77 (2002).

68. S. O. Kim, H. H. Solak, M. P. Stoykovich, N. J. Ferrier, J. J. de Pablo, and P. F. Nealey, Nature 424, 411 (2003).

69. N. P. Huang, R. Michel, J. Voros, M. Textor, R. Hofer, A. Rossi, D. L. Elbert, J. A. Hubbell, and N. D. Spencer, Langmuir 17, 489 (2001).

70. S. Pasche, S. M. De Paul, J. Voros, N. D. Spencer, and M. Textor, Langmuir 19, 9216 (2003).

71. G. L. Zhen, D. Falconnet, E. Kuennemann, J. Voros, N. Spencer, M. Textor, and S. Zuercher, Adv. Funct. Mater. (2005).

72. B. Stadler, D. Falconnet, I. Pfeiffer, F. Hook, and J. Voros, Langmuir 20, 11348 (2004).

73. B. Stadler, S. Frerker, H. Solak, and J. Voros, Nano Lett. (2005), in preparation.

74. B. Stadler, C. Huwiler, H. H. Solak, M. Textor, and J. Voros, Selective patterning and manipulation of gold colloids. in Proceedings of 2005 5th IEEE Conference on Nanotechnology, Nagoya, Japan (2005). 
75. Y. N. Xia, J. A. Rogers, K. E. Paul, and G. M. Whitesides, Chem. Rev. 99, 1823 (1999).

76. M. Brehmer, L. Conrad, and L. Funk, J. Dispersion Sci. Technol. 24, 291 (2003).

77. A. P. Quist, E. Pavlovic, and S. Oscarsson, Analytical and Bioanalytical Chemistry 381, 591 (2005).

78. A. Kumar and G. M. Whitesides, Appl. Phys. Lett. 63, 2002 (1993).

79. B. Michel, A. Bernard, A. Bietsch, E. Delamarche, M. Geissler, D. Juncker, H. Kind, J. P. Renault, H. Rothuizen, H. Schmid, P. Schmidt-Winkel, R. Stutz, and H. Wolf, IBM J. Res. Develop. 45, 697 (2001).

80. D. Stamou, C. Duschl, E. Delamarche, and H. Vogel, Angew. Chem.-Int. Ed. 42, 5580 (2003).

81. C. M. Niemeyer and C. A. Mirkin, Nanobiotechnology, Wiley$\mathrm{VCH}$, Weinheim (2004).

82. G. Csucs, R. Michel, J. W. Lussi, M. Textor, and G. Danuser, Biomaterials 24, 1713 (2003).

83. S. A. Lange, V. Benes, D. P. Kern, J. K. H. Horber, and A. Bernard, Anal. Chem. 76, 1641 (2004).

84. M. R. Dusseiller, D. Schlaepfer, M. Koch, R. Kroschewski, and M. Textor, Biomaterials 26, 5917 (2005).

85. G. Csucs, T. Kunzler, K. Feldman, F. Robin, and N. D. Spencer, Langmuir 19, 6104 (2003).

86. D. Lehnert, B. Wehrle-Haller, C. David, U. Weiland, C. Ballestrem, B. A. Imhof, and M. Bastmeyer, J. Cell Sci. 117, 41 (2004).

87. A. A. Tseng, K. Chen, C. D. Chen, and K. J. Ma, IEEE Transactions on Electronics Packaging Manufacturing 26, $1 \overline{41(\mathbf{2 0 0 3}) .}$

88. A. Bernard, J. P. Renault, B. Michel, H. R. Bosshard, and E. Delamarche, Adv. Mater. 12, 1067 (2000).

89. H. Schmid and B. Michel, Macromolecules 33, 3042 (2000).

90. A. Bietsch and B. Michel, J. Appl. Phys. 88, 4310 (2000).

91. J. C. Love, L. A. Estroff, J. K. Kriebel, R. G. Nuzzo, and G. M. Whitesides, Chem. Rev. 105, 1103 (2005).

92. K. E. Schmalenberg, H. M. Buettner, and K. E. Uhrich, Biomaterials 25, 1851 (2004).

93. Y. Xia, X.-M. Zhao, and G. M. Whitesides, Microelectronic Engineering 32, 255 (1996).

94. A. Bernard, E. Delamarche, H. Schmid, B. Michel, H. R. Bosshard, and H. Biebuyck, Langmuir 14, 2225 (1998).

95. J. P. Renault, A. Bernard, A. Bietsch, B. Michel, H. R. Bosshard, E. Delamarche, M. Kreiter, B. Hecht, and U. P. Wild, J. Phys. Chem. B 107, 703 (2003).

96. B. R. Young, W. G. Pitt, and S. L. Cooper, J. Colloid Interface Sci. 124, 28 (1988).

97. M. A. Markowitz, D. C. Turner, B. D. Martin, and B. P. Gaber, Appl. Biochem. Biotechnol. 68, 57 (1997).

98. M. Mayer, J. Yang, I. Gitlin, D. H. Gracias, and G. M. Whitesides, Proteomics 4, 2366 (2004)

99. J. L. Tan, J. Tien, and C. S. Chen, Langmuir 18, 519 (2002).

100. D. Mayer, D. Schwaab, O. Salomon, A. Offenhaeusser, A. Yasuda, and J. Wessels, In-situ Microcontact Printing of Proteins. AVS 52nd International Symposium (2005).

101. E. Delamarche, A. Bernard, H. Schmid, B. Michel, and H. Biebuyck, Science 276, 779 (1997).

102. E. Delamarche, H. Schmid, B. Michel, and H. Biebuyck, Adv. Mater. 9, 741 (1997).

103. I. Bohm, A. Lampert, M. Buck, F. Eisert, and M. Grunze, Appl. Surf. Sci. 141, 237 (1999).

104. K. Glasmastar, J. Gold, A. S. Andersson, D. S. Sutherland, and B. Kasemo, Langmuir 19, 5475 (2003).

105. D. J. Graham, D. D. Price, and B. D. Ratner, Langmuir 18, 1518 (2002).

106. J. A. Rogers, K. E. Paul, and G. M. Whitesides, J. Vac. Sci. Technol. B: Microelectronics and Nanometer Structures 16, 88 (1998).

107. A. A. Yu, T. A. Savas, G. S. Taylor, A. Guiseppe-Elie, H. I. Smith, and F. Stellacci, Nano Lett. 5, 1061 (2005).
108. T. A. Savas, M. L. Schattenburg, J. M. Carter, and H. I. Smith, J. Vac. Sci. Technol. B 14, 4167 (1996).

109. Y. Zhou, O. Andersson, P. Lindberg, and B. Liedberg, Microchimica Acta 146, 193 (2004).

110. S. Y. Chou, P. R. Krauss, and P. J. Renstrom, Appl. Phys. Lett. 67, 3114 (1995).

111. D. G. Choi, J. H. Jeong, Y. S. Sim, E. S. Lee, W. S. Kim, and B. S. Bae, Langmuir 21, 9390 (2005).

112. S. Zankovych, T. Hoffmann, J. Seekamp, J. U. Bruch, and C. M. S. Torres, Nanotechnology 12, 91 (2001).

113. C. M. S. Torres, S. Zankovych, J. Seekamp, A. P. Kam, C. C. Cedeno, T. Hoffmann, J. Ahopelto, F. Reuther, K. Pfeiffer, G. Bleidiessel, G. Gruetzner, M. V. Maximov, and B. Heidari, Materials Science \& Engineering C-Biomimetic and Supramolecular Systems 23, 23 (2003).

114. L. J. Guo, J. Phys. D-Appl. Phys. 37, R123 (2004).

115. S. Park, S. Saxer, C. Padeste, H. H. Solak, J. Gobrecht, and H. Schift, Microelectronic Engineering 78-79, 682 (2005).

116. E. Kim, Y. N. Xia, X. M. Zhao, and G. M. Whitesides, $A d v$. Mater. 9, 651 (1997).

117. M. Colburn, S. Johnson, M. Stewart, S. Damle, B. Bailey, B. Choi, M. Wedlake, T. Michaelson, S. V. Sreenivasan, J. G. Ekerdt, and C. G. Willson, Proc. SPIE-Int. Soc. Opt. Eng. 3676, 379 (1999).

118. L. Tan, Y. P. Kong, S. W. Pang, and A. F. Yee, J. Vac. Sci. Technol. B 22, 2486 (2004).

119. J. N. Lee, C. Park, and G. M. Whitesides, Anal. Chem. 75, 6544 (2003).

120. D. J. Resnick, S. V. Sreenivasan, and C. G. Willson, MaterialsToday (2005).

121. A. Lebib, Y. Chen, J. Bourneix, F. Carcenac, E. Cambril, L. Couraud, and H. Launois, Microelectronic Engineering 46, 319 (1999)

122. T. Haatainen and J. Ahopelto, Physica Scripta 67, 357 (2003).

123. H. Tan, A. Gilbertson, and S. Y. Chou, J. Vac. Sci. Technol. B 16 , 3926 (1998).

124. J. Wang, X. Y. Sun, L. Chen, and S. Y. Chou, Appl. Phys. Lett. 75 , 2767 (1999).

125. L. J. Guo, X. Cheng, and C. F. Chou, Nano Lett. 4, 69 (2004).

126. R. Bunk, P. Carlberg, A. Mansson, I. A. Nicholls, P. Omling, M. Sundberg, S. Tagerud, and L. Montelius, Japanese Journal of Applied Physics Part 1-Regular Papers Brief Communications \& Review Papers 44, 3337 (2005).

127. A. Pepin, P. Youinou, V. Studer, A. Lebib, and Y. Chen, Microelectronic Engineering 61-2, 927 (2002).

128. B. G. Casey, D. R. S. Cumming, I. I. Khandaker, A. S. G. Curtis, and C. D. W. Wilkinson, Microelectronic Engineering 46, 125 (1999).

129. M. J. Dalby, N. Gadegaard, M. O. Riehle, C. D. W. Wilkinson, and A. S. G. Curtis, Int. J. Biochem. Cell Biol. 36, 2005 (2004).

130. E. K. F. Yim, R. M. Reano, S. W. Pang, A. F. Yee, C. S. Chen, and K. W. Leong, Biomaterials 26, 5405 (2005).

131. F. Matsumoto, K. Nishio, T. Miyasaka, and H. Masuda, Jpn. J. Appl. Phys. Part 2-Letters \& Express Letters 43, L640 (2004).

132. J. D. Hoff, L. J. Cheng, E. Meyhofer, L. J. Guo, and A. J. Hunt, Nano Lett. 4, 853 (2004).

133. T. Ohtake, K. Nakamatsu, S. Matsui, H. Tabata, and T. Kawai, J. Vac. Sci. Technol. B 22, 3275 (2004).

134. D. Falconnet, D. Pasqui, S. Park, R. Eckert, H. Schift, J. Gobrecht, R. Barbucci, and M. Textor, Nano Lett. 4, 1909 (2004).

135. D. J. Wales, Science $271,925(\mathbf{1 9 9 6 )}$.

136. F. Sun, W. Cai, Y. Li, G. Duan, W. T. Nichols, C. Liang, N. Koshizaki, Q. Fang, and I. W. Boyd, Appl. Phys. B: Lasers and Optics 81, 765 (2005)

137. C. L. Haynes and R. P. Van Duyne, J. Phys. Chem. B 105, 5599 (2001).

138. A. N. Shipway, E. Katz, and I. Willner, Chem. Phys. Chem. 1, 18 (2000). 
139. H. W. Deckman and J. H. Dunsmuir, Appl. Phys. Lett. 41, 377 (1982).

140. J. C. Hulteen and R. P. Van Duyne, J. Vac. Sci. Technol. A: Vacuum, Surfaces, and Films 13, 1553 (1995).

141. F. Burmeister, W. Badowsky, T. Braun, S. Wieprich, J. Boneberg, and P. Leiderer, Appl. Surf. Sci. 145, 461 (1999).

142. X. D. Wang, C. J. Summers, and Z. L. Wang, Nano Lett. 4, 423 (2004).

143. Y. A. Vlasov, X. Z. Bo, J. C. Sturm, and D. J. Norris, Nature 414, 289 (2001)

144. K. P. Velikov, C. G. Christova, R. P. A. Dullens, and A. van Blaaderen, Science 296, 106 (2002).

145. F. Zeng, Z. W. Sun, C. Y. Wang, B. Y. Ren, X. X. Liu, and Z. Tong, Langmuir 18, 9116 (2002).

146. Y. X. Zhao, K. Wostyn, G. de Schaetzen, K. Clays, L. Hellemans, A. Persoons, M. Szekeres, and R. A. Schoonheydt, Appl. Phys. Lett. 82, 3764 (2003)

147. Y. D. Yin and Y. N. Xia, Adv. Mater. 13, 267 (2001).

148. J. Tien, A. Terfort, and G. M. Whitesides, Langmuir 13, 5349 (1997).

149. Y. J. Sun and G. C. Walker, J. Phys. Chem. B 106, 2217 (2002)

150. U. Jonas, A. del Campo, C. Kruger, G. Glasser, and D. Boos, Proceedings of the National Academy of Sciences of the United States of America 99, 5034 (2002).

151. V. Santhanam and R. P. Andres, Nano Lett. 4, 41 (2004).

152. C. Huwiler, M. Halter, K. Rezwan, D. Falconnet, M. Textor, and J. Voeroes, Nanotechnology 16, 3045 (2005).

153. F. Juillerat, H. H. Solak, P. Bowen, and H. Hofmann, Nanotechnology 16, 1311 (2005).

154. D. Qin, Y. N. Xia, B. Xu, H. Yang, C. Zhu, and G. M. Whitesides, Adv. Mater. 11, 1433 (1999).

155. Y. Cui, M. T. Bjork, J. A. Liddle, C. Sonnichsen, B. Boussert, and A. P. Alivisatos, Nano Lett. 4, 1093 (2004)

156. D. Xia and S. R. J. Brueck, Nano Lett. (2004)

157. I. Lee, H. P. Zheng, M. F. Rubner, and P. T. Hammond, Adv. Mater 14, 572 (2002).

158. U. C. Fischer and H. P. Zingsheim, J. Vac. Sci. Technol. 19, 881 (1981).

159. R. Michel, I. Reviakine, D. Sutherland, C. Fokas, G. Csucs, G. Danuser, N. Spencer, and M. Textor, Langmuir 18, 8580 (2002).

160. P. Hanarp, D. S. Sutherland, J. Gold, and B. Kasemo, Colloids and Surfaces A: Physicochemical and Engineering Aspects $\overline{214,23}$ (2003).

161. E. Snoeks, A. van Blaaderen, T. van Dillen, C. M. van Kats, K. Velikov, M. L. Brongersma, and A. Polman, Nuclear Instruments \& Methods in Physics Research Section B-Beam Interactions with Materials and Atoms 178, 62 (2001).

162. J. C. Garno, N. A. Amro, K. Wadu-Mesthrige, and G. Y. Liu, Langmuir 18, 8186 (2002).

163. A. J. Haes and R. P. Van Duyne, J. Am. Chem. Soc. 124, 10596 (2002).

164. A. J. Haes, W. P. Hall, L. Chang, W. L. Klein, and R. P. VanDuyne, Nano Lett. 4, 1029 (2004).

165. A. J. Haes, S. Zou, G. C. Schatz, and R. P. VanDuyne, J. Phys. Chem. B 108, 6961 (2004).

166. A. B. Kharitonov, A. N. Shipway, and I. Willner, Anal. Chem. 71, 5441 (1999).

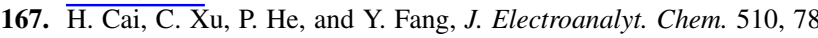
(2001).

168. S. Schultz, D. R. Smith, J. J. Mock, and D. A. Schultz, Proceedings of the National Academy of Sciences of the United States of America 97, 996 (2000).

169. R. Moller, A. Csaki, J. M. Kohler, and W. Fritzsche, Nucl. Acids Res. 28, e91 (2000)

170. O. D. Velev and E. W. Kaler, Langmuir 15, 3693 (1999).

171. R. Moller, A. Csaki, J. M. Kohler, and W. Fritzsche, Langmuir 17 , 5426 (2001)
172. S.-J. Park, A. A. Lazarides, C. A. Mirkin, P. W. Brazis, C. R. Kannewurf, and R. L. Letsinger, Angew. Chem. 39, 3845 (2000).

173. W. Yang, D. W. Trau, R. Renneberg, N. T. Yu, and F. Caruso, J. Colloid Interface Sci. 234, 356 (2001).

174. N. D. Denkov, O. D. Velev, P. A. Kralchevsky, I. B. Ivanov, H. Yoshimura, and K. Nagayama, Nature 361, 26 (1993).

175. N. D. Denkov, O. D. Velev, P. A. Kralchevsky, I. B. Ivanov, H. Yoshimura, and K. Nagayama, Langmuir 8, 3183 (1992).

176. J. C. Hulteen, D. A. Treichel, M. T. Smith, M. L. Duval, T. R. Jensen, and R. P. Van Duyne, J. Phys. Chem. B 103, 3854 (1999).

177. F. Burmeister, C. Schafle, T. Matthes, M. Bohmisch, J. Boneberg, and P. Leiderer, Langmuir 13, 2983 (1997).

178. A. D. Ormonde, D. Erin, M. Hicks, J. Castillo, and R. P. van Dyne, Langmuir 20, 6927 (2004).

179. C. L. Haynes, A. D. McFarland, M. T. Smith, J. C. Hulteen, and R. P. Van Duyne, J. Phys. Chem. B 106, 1898 (2002).

180. P. Mulvaney, Langmuir 12,788 (1996).

181. M. J. Feldstein, C. D. Keating, Y. H. Liau, M. J. Natan, and N. F. Scherer, J. Am. Chem. Soc. 119, 6638 (1997).

182. J. Shi, S. Gider, K. Babcock, and D. D. Awschalom, Science 271, 937 (1996)

183. R. P. Andres, J. D. Bielefeld, J. I. Henderson, D. B. Janes, V. R. Kolagunta, C. P. Kubiak, W. J. Mahoney, and R. G. Osifchin, Science 273, 1690 (1996).

184. F. Frederix, J. M. Friedt, K. H. Choi, W. Laureyn, A. Campitelli, D. Mondelaers, G. Maes, and G. Borghs, Anal. Chem. 75, 6894 (2003).

185. J. C. Riboh, A. J. Haes, A. D. McFarland, C. Ranjit Yonzon, and R. P. Van Duyne, J. Phys. Chem. B 107, 1772 (2003).

186. A.-S. Andersson, F. Backhed, A. von Euler, A. Richter-Dahlfors, D. Sutherland, and B. Kasemo, Biomaterials 24, 3427 (2003).

187. M. Breulmann, S. Forster, and M. Antonietti, Macromol. Chem. Phys. 201, 204 (2000)

188. J. K. Cox, A. Eisenberg, and R. B. Lennox, Current Opinion in Colloid \& Interface Science 4, 52 (1999).

189. J. C. Meiners, H. Elbs, A. Ritzi, J. Mlynek, and G. Krausch, J. Appl. Phys. 80, 2224 (1996).

190. B. H. Sohn, S. I. Yoo, B. W. Seo, S. H. Yun, and S. M. Park, J. Am. Chem. Soc. 123, 12734 (2001).

191. J. P. Spatz, S. Sheiko, and M. Moller, Macromolecules 29, 3220 (1996).

192. J. P. Spatz, S. Mossmer, and M. Moller, Chemistry-A European Journal 2, 1552 (1996)

193. R. Glass, M. Arnold, J. Blummel, A. Kuller, M. Moller, and J. P. Spatz, Adv. Funct. Mater. 13, 569 (2003).

194. J. P. Spatz, V. Z. H. Chan, S. Mossmer, F. M. Kamm, A. Plettl, P. Ziemann, and M. Moller, Adv. Mater. 14, 1827 (2002).

195. A. Golzhauser, W. Eck, W. Geyer, V. Stadler, T. Weimann, P. Hinze, and M. Grunze, Adv. Mater. 13, 806 (2001)

196. J. P. Spatz, S. Mossmer, C. Hartmann, M. Moller, T. Herzog, M. Krieger, H. G. Boyen, P. Ziemann, and B. Kabius, Langmuir 16, 407 (2000)

197. R. Glass, M. Moller, and J. P. Spatz, Nanotechnology 14, 1153 (2003).

198. C. Minelli, C. Hinderling, H. Heinzelmann, R. Pugin, and M. Liley, Langmuir 21, 7080 (2005).

199. C. Hinderling, Y. Keles, T. Stockli, H. E. Knapp, T. de los Arcos, P. Oelhafen, I. Korczagin, M. A. Hempenius, G. J. Vancso, R. L. Pugin, and H. Heinzelmann, Adv. Mater. 16, 876 (2004).

200. M. Aizawa and A. M. Buriak, J. Am. Chem. Soc. 127, 8932 (2005)

201. P. Mansky, C. K. Harrison, P. M. Chaikin, R. A. Register, and N. Yao, Appl. Phys. Lett. 68, 2586 (1996).

202. J. P. Spatz, T. Herzog, S. Mossmer, P. Ziemann, and M. Moller, Adv. Mater. 11, 149 (1999). 
203. N. Kumar and J. I. Hahm, Langmuir 21, 6652 (2005).

204. Y. S. Seo, H. Luo, V. A. Samuilov, M. H. Rafailovich, J. Sokolov, D. Gersappe, and B. Chu, Nano Lett. 4, 659 (2004)

205. R. Glass, M. Arnold, E. A. Cavalcanti-Adam, J. Blummel, C. Haferkemper, C. Dodd, and J. P. Spatz, New J. Phys. 6, (2004).

206. J. Brugger, J. W. Berenschot, S. Kuiper, W. Nijdam, B. Otter, and M. Elwenspoek, Microelectronic Engineering 53, 403 (2000).

207. G. M. Kim, M. A. F. van den Boogaart, and J. Brugger, Microelectronic Engineering 67-8, 609 (2003).

208. A. Tixier, Y. Mita, J. P. Gouy, and H. Fujita, J. Micromechan. Microengineer. 10, 157 (2000).

209. K. Ono, H. Shimada, S. I. Kobayashi, and Y. Ootuka, Jpn. J. Appl. Phys. Part 1-Regular Papers Short Notes \& Review Papers 35, 2369 (1996).

210. C. Stamm, F. Marty, A. Vaterlaus, V. Weich, S. Egger, U. Maier, U. Ramsperger, H. Fuhrmann, and D. Pescia, Science 282, 449 (1998).

211. G. M. Kim, S. Kawai, M. Nagashio, H. Kawakatsu, and J. Brugger, J. Vac. Sci. Technol. B 22, 1658 (2004).

212. P. Zahl, M. Bammerlin, G. Meyer, and R. R. Schlittler, Rev. Sci. Instrum. 76 (2005).

213. R. Luthi, R. R. Schlittler, J. Brugger, P. Vettiger, M. E. Welland, and J. K. Gimzewski, Appl. Phys. Lett. 75, 1314 (1999).

214. C. V. Cojocaru, C. Harnagea, F. Rosei, A. Pignolet, M. A. F. van den Boogaart, and J. Brugger, Appl. Phys. Lett. 86 (2005).

215. A. Ludwig, J. Cao, J. Brugger, and I. Takeuchi, Measurement Science \& Technology 16, 111 (2005).

216. M. Kolbel, R. W. Tjerkstra, J. Brugger, C. J. M. van Rijn, W. Nijdam, J. Huskens, and D. N. Reinhoudt, Nano Lett. 2, 1339 (2002).

217. V. Bucher, J. Brugger, D. Kern, G. M. Kim, M. Schubert, and W. Nisch, Microelectronic Engineering 61-2, 971 (2002)

218. D. V. Nicolau, T. Taguchi, H. Taniguchi, and S. Yoshikawa, Lang muir 15, 3845 (1999)

219. G. J. Zhang, T. Tanii, T. Zako, T. Hosaka, T. Miyake, Y. Kanari, T. W. Funatsu, and I. Ohdomari, Small 1, 833 (2005).

220. J. Lussi, C. Tang, P.-A. Kuenzi, U. Staufer, G. Csucs, J. Voeroes, G. Danuser, J. A. Hubbel, and M. Textor, Nanotechnology 16, 1781 (2005).

221. T. R. Groves, D. Pickard, B. Rafferty, N. Crosland, D. Adam, and G. Schubert, Microelectronic Engineering 61-2, 285 (2002).

222. A. A. Tseng, Small 1, 924 (2005).

223. G. J. Leggett, Analyst 130, 259 (2005).

224. R. D. Piner, J. Zhu, F. Xu, S. H. Hong, and C. A. Mirkin, Science 283, 661 (1999)

225. S. H. Hong, J. Zhu, and C. A. Mirkin, Science 286, 523 (1999).

226. S. Hong and C. A. Mirkin, Science 288, 1808 (2000).

227. S. Rozhok, R. Piner, and C. A. Mirkin, J. Phys. Chem. B 107, 751 (2003).

228. C. A. Mirkin, S. Hong, and L. M. Demers, Chem. Phys. Chem. 2 , 37 (2001).

229. P. V. Schwartz, Langmuir 18, 4041 (2002).

230. P. E. Sheehan and L. J. Whitman, Phys. Rev. Lett. 88, 156104 (2002).

231. P. Manandhar, J. Jang, G. C. Schatz, M. A. Ratner, and S. Hong, Phys. Rev. Lett. 90, 115505 (2003).

232. J. Jang, S. Hong, G. C. Schatz, and M. A. Ratner, J. Chem. Phys. 115, 2721 (2001).

233. B. L. Weeks, A. Noy, A. E. Miller, and J. J. De Yoreo, Phys. Rev. Lett. 88, 255505 (2002).

234. K. Salaita, S. W. Lee, X. F. Wang, L. Huang, T. M. Dellinger, C. Liu, and C. A. Mirkin, Small 1, 940 (2005).
235. X. Wang and C. Liu, Nano Lett. 5, 1867 (2005).

236. M. Su and V. P. Dravid, Appl. Phys. Lett. 80, 4434 (2002).

237. R. McKendry, W.T. S. Huck, B. Weeks, M. Fiorini, C. Abell, and T. Rayment, Nano Lett. 2, 713 (2002).

238. M. Su, M. Aslam, L. Fu, N. Q. Wu, and V. P. Dravid, Appl. Phys. Lett. 84, 4200 (2004).

239. M. Ben Ali, T. Ondarcuhu, M. Brust, and C. Joachim, Langmuir 18, 872 (2002).

240. P. J. Thomas, G. U. Kulkarni, and C. N. R. Rao, J. Mater. Chem. 14,625 (2004)

241. Y. Li, B. W. Maynor, and J. Liu, J. Am. Chem. Soc. 123, 2105 (2001).

242. B. W. Maynor, Y. Li, and J. Liu, Langmuir 17, 2575 (2001).

243. B. W. Maynor, J. Li, C. Lu, and J. Liu, J. Am. Chem. Soc. 126, 6409 (2004).

244. D. A. Weinberger, S. Hong, C. A. Mirkin, B. W. Wessels, and T. B. Higgins, Adv. Mater. 12, 1600 (2000).

245. D. L. Wilson, R. Martin, S. Hong, M. Cronin-Golomb, C. A. Mirkin, and D. L. Kaplan, Proceedings of the National Academy of Sciences of the United States of America 98, 13660 (2001)

246. G. Agarwal, R. R. Naik, and M. O. Stone, J. Am. Chem. Soc. 125 , 7408 (2003).

247. G. Agarwal, L. A. Sowards, R. R. Naik, and M. O. Stone, J. Am. Chem. Soc. 125, 580 (2003).

248. L. M. Demers, S. J. Park, T. A. Taton, Z. Li, and C. A. Mirkin, Angew. Chem.-Int. Ed. 40, 3071 (2001).

249. L. M. Demers, D. S. Ginger, S. J. Park, Z. Li, S. W. Chung, and C. A. Mirkin, Science 296, 1836 (2002).

250. D. Nyamjav and A. Ivanisevic, Adv. Mater. 15, 1805 (2003).

251. K. B. Lee, S. J. Park, C. A. Mirkin, J. C. Smith, and M. Mrksich, Science 295, 1702 (2002).

252. J. Hyun, S. J. Ahn, W. K. Lee, A. Chilkoti, and S. Zauscher, Nano Lett. 2, 1203 (2002)

253. K. B. Lee, J. H. Lim, and C. A. Mirkin, J. Am. Chem. Soc. 125 , 5588 (2003).

254. D. J. Pena, M. P. Raphael, and J. M. Byers, Langmuir 19, 9028 (2003).

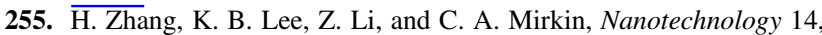
1113 (2003).

256. J. H. Lim, D. S. Ginger, K. B. Lee, J. Heo, J. M. Nam, and C. A. Mirkin, Angew. Chem.-Int. Ed. 42, 2309 (2003).

257. A. Noy, A. E. Miller, J. E. Klare, B. L. Weeks, B. W. Woods, and J. J. DeYoreo, Nano Lett. 2, 109 (2002)

258. S. Xu and G. Y. Liu, Langmuir 13, 127 (1997).

259. K. Wadu-Mesthrige, S. Xu, N. A. Amro, and G. Y. Liu, Langmuir 15,8580 (1999).

260. G. Y. Liu, S. Xu, and Y. Qian, Acc. Chem. Res. 33, 457 (2000).

261. K. Wadu-Mesthrige, N. A. Amro, and G. Y. Liu, Scanning 22, 380 (2000).

262. K. Wadu-Mesthrige, N. A. Amro, J. C. Garno, S. Xu, and G. Y. Liu, Biophysical J. 80, 1891 (2001).

263. J. F. Liu, S. Cruchon-Dupeyrat, J. C. Garno, J. Frommer, and G. Y. Liu, Nano Lett. 2, 937 (2002).

264. N. A. Amro, S. Xu, and G. Y. Liu, Langmuir 16, 3006 (2000).

265. G.-Y. Liu and N. A. Amro, PNAS 99, 5165 (2002).

266. C. K. Harnett, K. M. Satyalakshmi, and H. G. Craighead, Langmuir 17,178 (2001)

267. H. H. Solak and C. David, J. Vac. Sci. Technol. B 21, 2883 (2003).

268. G.-J. Zhang, T. T., T. Zako, H. T., M. T., Y. Kanari, T. Funatsu, and I. Ohdomari, Small 1, 833 (2005). 